University of Rhode Island

DigitalCommons@URI

Open Access Master's Theses

2014

\title{
THREE-DIMENSIONAL TOPOLOGY OPTIMIZATION OF STATICALLY LOADED POROUS AND MULTI-PHASE STRUCTURES
}

Matthew Okruta

University of Rhode Island, matthew_okruta@my.uri.edu

Follow this and additional works at: https://digitalcommons.uri.edu/theses

\section{Recommended Citation}

Okruta, Matthew, "THREE-DIMENSIONAL TOPOLOGY OPTIMIZATION OF STATICALLY LOADED POROUS AND MULTI-PHASE STRUCTURES" (2014). Open Access Master's Theses. Paper 347.

https://digitalcommons.uri.edu/theses/347

This Thesis is brought to you for free and open access by DigitalCommons@URI. It has been accepted for inclusion in Open Access Master's Theses by an authorized administrator of DigitalCommons@URI. For more information, please contact digitalcommons-group@uri.edu. 
THREE-DIMENSIONAL TOPOLOGY OPTIMIZATION OF STATICALLY

LOADED POROUS AND MULTI-PHASE STRUCTURES

BY

MATTHEW OKRUTA

A THESIS SUBMITTED IN PARTIAL FULFILLMENT OF THE

REQUIREMENTS FOR THE DEGREE OF

MASTERS OF SCIENCE

IN

MECHANICAL ENGINEERING AND APPLIED MECHANICS

UNIVERSITY OF RHODE ISLAND

2014 
MASTER OF SCIENCE THESIS

OF

MATTHEW OKRUTA

APPROVED:

Thesis Committee:

Major Professor David G. Taggart

Carl-Ernst Rousseau

Frederick J. Vetter

Nasser H. Zawia

DEAN OF THE GRADUATE SCHOOL

UNIVERSITY OF RHODE ISLAND

2014 


\begin{abstract}
A novel topology optimization method called Prescribed Material

Redistribution (PMR) has been under development at the University of Rhode Island for the past several years. Originally implemented through a series of Fortran subroutines used in conjunction with the commercial finite element package, Abaqus, a standalone two-dimensional Matlab code was developed and used in evaluating the method. In order to explore the capabilities of the PMR scheme for three-dimensional problems, it became necessary to develop a three-dimensional version of the PMR Matlab code.
\end{abstract}

The objective of this thesis, therefore, is the development and evaluation of a three-dimensional Matlab implementation of the PMR scheme. The code allows users to analyze general topology optimization problems by defining an appropriate design domain, load conditions, support conditions, predefined fully dense or void regions, and symmetry conditions. The code also provides the capability to impose constraint conditions where coupling of displacement degrees of freedoms can be specified by the user. A primary aspect of this work is the development and implementation of hexahedral finite element equations. Since three-dimensional problems can be computationally intensive, the finite element analysis implementation includes computationally efficient algorithms where feasible. The post-processing phase of the analysis included the generation of optimized three-dimensional geometry in the standard STL file format. The STL file allows users to examine the results using standard CAD file viewers. Also, the STL file can be used as input to additive 
manufacturing equipment such as 3-D printing for the manufacture of physical components.

The three-dimensional PMR code is evaluated by two types of optimization problems. The first set of test cases investigated are based on the identification of a known topology for a centrally loaded, simply supported beam. Although this problem can be considered using a two-dimensional analysis, performing a threedimensional analysis allowed for the ability to consider several different symmetry cases. This is useful for evaluation of the symmetry capabilities of the threedimensional PMR code. For the symmetry case where all three coordinate axes define symmetry place, an alternate test case was developed and used for evaluating the code. The second set of test cases were designed to identify optimal topologies for twophase composite microstructures under general three-dimensional stress states. By defining unit cell models with appropriate loading and constraint conditions, any three-dimensional stress states can be modeled. If one of the composite phases is taken to have essentially zero stiffness, this approach can be used to determine the optimized microstructure of porous materials. Several test cases are evaluated for the identification of optimized microstructures for both porous and composite materials. 


\section{ACKNOWLEDGEMENTS}

Foremost, I would like to express my sincere gratitude to my graduate advisor Professor David Taggart for the continuous support of my Master's Thesis research over the past two years. His willingness to provide assistance at all times and understand my struggles throughout the journey are greatly appreciated, and I could not have asked for a better advisor and mentor.

I would also like to thank the professors on my Thesis Committee for volunteering their own valuable time towards helping me complete my defense. These individuals include Professor Carl-Ernst Rousseau, Professor Frederick Vetter, and Professor Aaron Bradshaw.

And lastly I would like to thank my loving family for staying by my side the entire time as I complete my graduate degree. 


\section{TABLE OF CONTENTS}

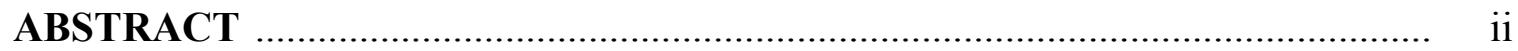

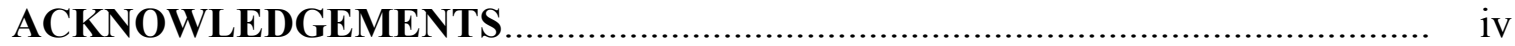

TABLE OF CONTENTS

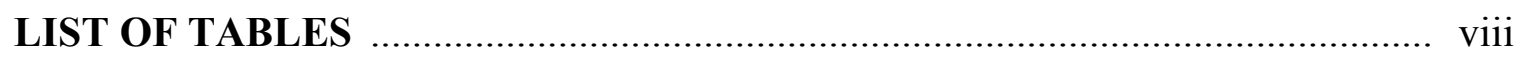

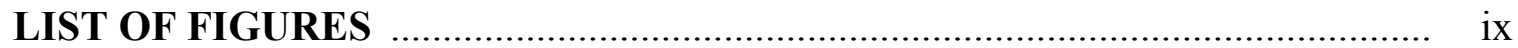

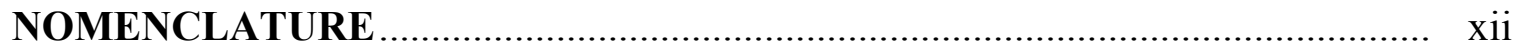

\section{CHAPTER 1 - INTRODUCTION}

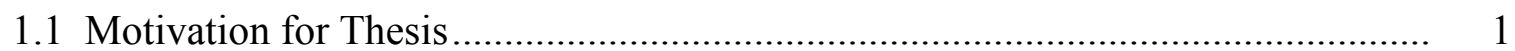

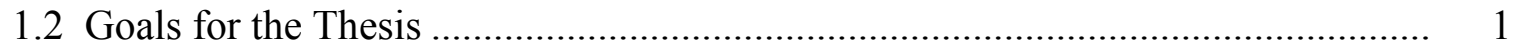

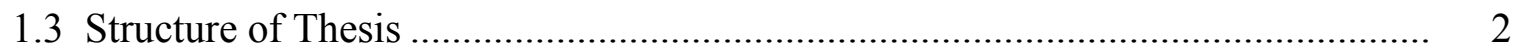

\section{CHAPTER 2 - TOPOLOGY OPTIMIZATION}

2.1 Optimization History ...................................................................................... 5

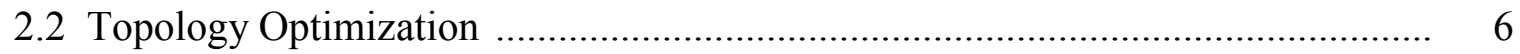

2.3 Homogenization Based Optimization ................................................................. 7

2.4 Solid Isotropic Material with Penalization .......................................................... 8

2.5 ESO Algorithms ………................................................................... 10

2.5.1 Evolutionary Structural Optimization ………............................................. 11 
2.5.2 Bi-Directional Evolutionary Structural Optimization

2.6 Numerical Problems

2.6.1 Checkerboarding

2.6.2 Mesh Dependence

2.7 Prescribed Material Redistribution 16

\section{CHAPTER 3 - FINITE ELEMENT ANALYSIS}

3.1 Three-Dimensional Elements

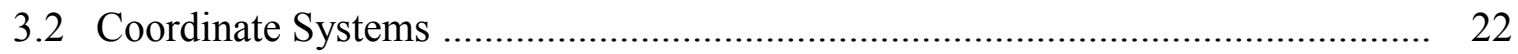

3.2.1 Local Coordinate System ……………………...................................... 23

3.2.2 Global Coordinate System ……................................................................ 25

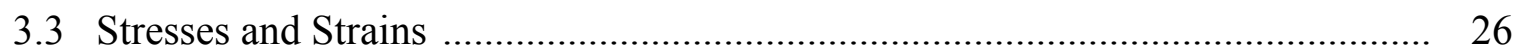

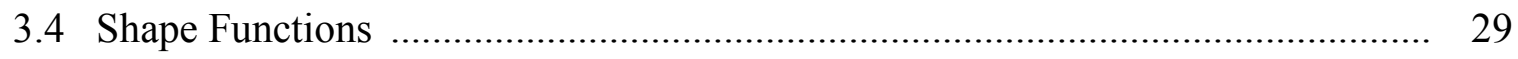

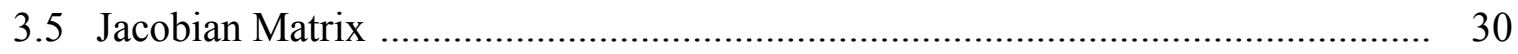

3.6 Strain-Displacement Matrix …………………....................................... 30

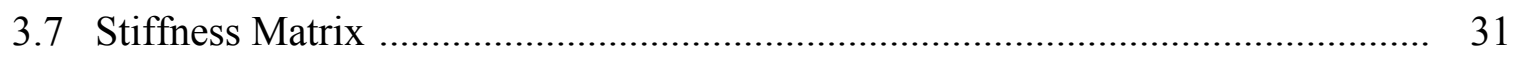

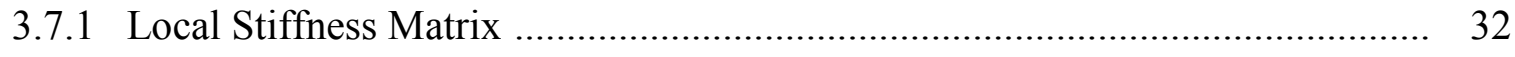

3.7.2 Global Stiffness Matrix ……………………......................................... 32

3.8 Stress and Strain Calculations ....................................................................... 33 


\section{CHAPTER 4 - THREE-DIMENSIONAL PMR CODE DEVELOPMENT}

4.1 Matlab Introduction .............................................................................. 34

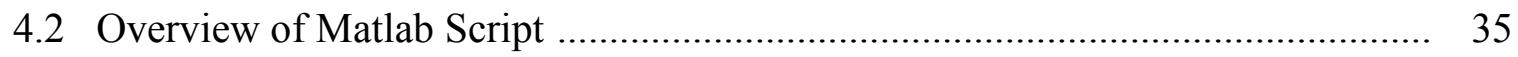

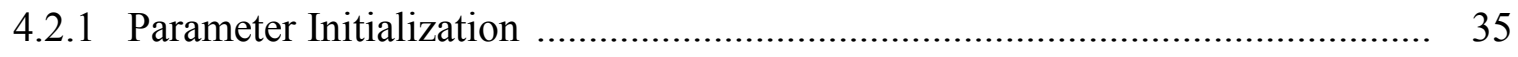

4.2.2 Finite Element Function ....................................................................... 39

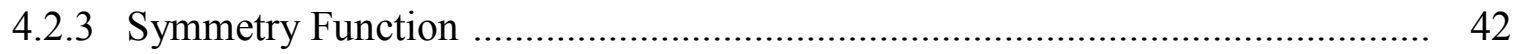

4.3 STL File Generation ........................................................................ 44

\section{CHAPTER 5 - ANALYSIS OF RESULTS}

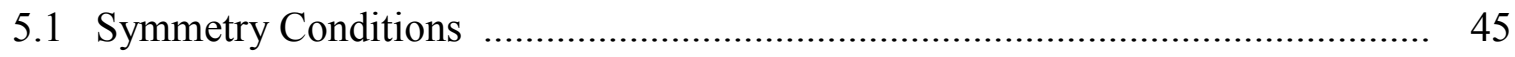

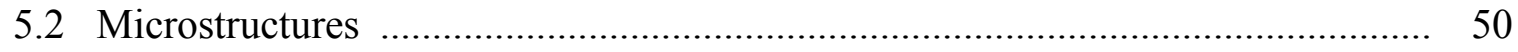

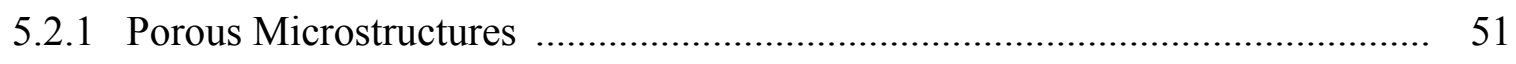

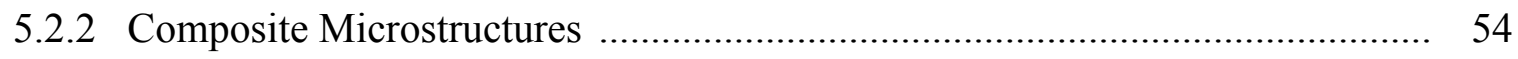

\section{CHAPTER 6 - CONCLUSIONS AND FUTURE WORK}

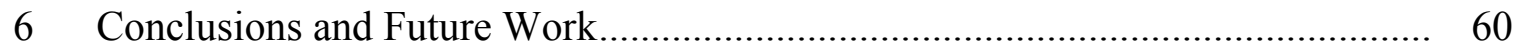

APPENDIX A - SYMMETRY TEST CASES ............................................ 62

APPENDIX B - MATLAB SCRIPTS FOR SYMMETRY CASES .................... 64

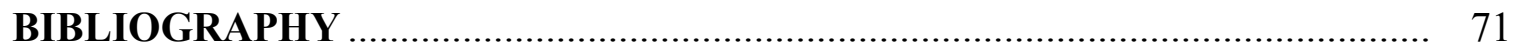




\section{LIST OF TABLES}

Table 3.1 Node numbering and coordinate locations for local hexahedral element ...25

Table 4.1 Predefined regions with corresponding node type and node densities .......36

Table 4.2 Matlab code translations for FE section ................................................. 39

Table 4.3 Matlab translations and corresponding equations/tables for FE function ... 40

Table 5.1 PMR and STL time results for similar example using all eight symmetry

cases

Table 5.2 Total CPU-time results for similar unique example using no symmetry (Case 8A) and full symmetry (Case 8B and Case 8C)........................... 48

Table 5.3 Stress values for each porous microstructure figure .............................51

Table 5.4 Stress values for each composite microstructure figure ............................. 55 


\section{LIST OF FIGURES}

Figure 2.1 Examples of (a) Size Optimization, (b) Shape Optimization and

(c) Topology Optimization [9] ..................................................

Figure 2.2 Illustration of composite material made of a periodic

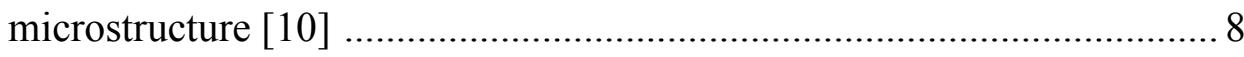

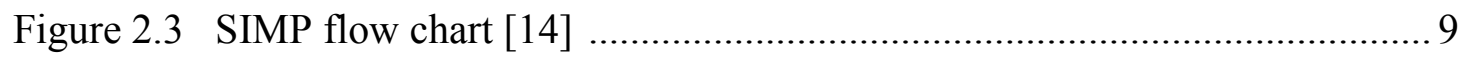

Figure 2.4 A catenary-type structure (a) Initial design (b)-(d) Evolution of optimal shape [17] 11

Figure 2.5 (a) Design case, (b) Checkerboard example, (c) Solution for 600

element discretization, (d) Solution for 5400 element discretization,

(e) Non-unique example [9] 13

Figure 2.6 Transition from initial to final relative density distribution for the case

$\rho_{0}=0.3[4]$ 18

Figure 2.7 Transition from initial to final cumulative relative density distribution for

the case $\rho_{0}=0.3[4]$ 18

Figure 3.1 Three common element types in three-dimensional analysis [24] 21

Figure 3.2 Local coordinate system for a hexahedral element with appropriate nodal numbering 24 
Figure 3.3 Global coordinate system for multiple hexahedral elements with

appropriate nodal numbering. 26

Figure 3.4 Three-dimensional element under a state of stress [27] 27

Figure 5.1 Theoretical optimal Michell-Arch structure 46

Figure 5.2 Michell-arch results using xy-symmetry for Case 6B.....

Figure 5.3 Initial conditions for Case 8 with full symmetry 48

Figure 5.4 Full symmetry test cases results: Case 8A (top), Case 8 (middle)

and $8 \mathrm{C}$ (bottom)

Figure 5.5 Outline of unit cell model region 50

Figure 5.6 Unit cell model with constraints shown 51

Figure 5.7 Microstructures made of porous materials; (a) through (h) cases correspond to conditions in Table 5.3 52

Figure 5.8 Two-phase composite microstructures; (a) through (g) cases correspond to conditions in Table 5.4 55

Figure 5.9 3-D printed physical model of case shown in Figure 5.7(c) 58

Figure 5.10 3-D printed physical model of case shown in Figure 5.7(d) 58

Figure A.1 Michell-arch results using no symmetry for Case 1 62

Figure A.2 Michell-arch results using x-symmetry for Case 2 62 


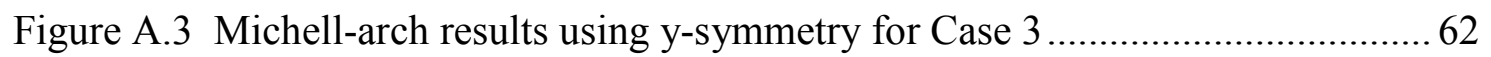

Figure A.4 Michell-arch results using z-symmetry for Case 4 ................................63

Figure A.5 Michell-arch results using xy-symmetry for Case 5 .............................63

Figure A.6 Michell-arch results using zx-symmetry for Case 6 .............................63

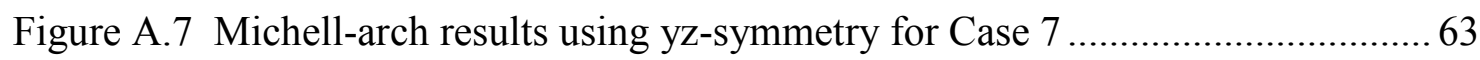




\section{NOMENCLATURE}

\section{Acronyms:}

AESO Additive Evolutionary Structural Optimization

$\mathrm{AR}$

Admission Volume Ratio

$\mathrm{BC}$

Boundary Condition

BESO Bidirectional Evolutionary Structural Optimization

CPU Central Processing Unit

DOF Degree of Freedom

ER Evolutionary Ratio

ESO Evolutionary Structural Optimization

FE Finite Element

FEA Finite Element Analysis

FEM Finite Element Method

LENS Laser Engineered Net Shaping

MRT Maxwell Reciprocal Theorem

PDE Partial Differential Equation

PMR Prescribed Material Redistribution

SIMP Solid Isotropic Material with Penalization 
STL Standard Tessellation Language

\section{Symbols}

\begin{tabular}{|c|c|}
\hline $\mathrm{r}, \mathrm{s}$ & Beta distribution parameters (Ch. 2) \\
\hline$\beta$ & Beta function \\
\hline$\beta_{\text {inc }}$ & incomplete Beta function \\
\hline$x, y, z$ & global coordinate notation \\
\hline$s, t, r$ & local coordinate notation $(\mathrm{Ch} .3)$ \\
\hline$z^{\prime}$ & local coordinate notation used by Logan (respective to $z$ in global) \\
\hline$\delta$ & Delta function \\
\hline$u, v, w$ & displacement parameters \\
\hline$[C]$ & elasticity matrix \\
\hline$\Gamma$ & Gamma function \\
\hline $\mathrm{H}$ & Heaviside step function \\
\hline$[J]$ & Jacobian \\
\hline$J$ & determinant of Jacobian \\
\hline$[J]^{-1}$ & inverse Jacobian \\
\hline$\rho$ & relative density \\
\hline$\rho_{0}$ & initial relative densit \\
\hline
\end{tabular}




$\begin{array}{ll}\rho_{\text {min }} & \text { minimum relative density } \\ N & \text { shape function } \\ N_{i} & \text { shape function for } i^{\text {th }} \text { node } \\ \varepsilon_{x}, \varepsilon_{y}, \varepsilon_{z} & \text { normal strains } \\ \gamma_{x y}, \gamma_{y z}, \gamma_{z x} & \text { shear strains } \\ {[B]} & \text { strain-displacement matrix } \\ \sigma_{x}, \sigma_{y}, \sigma_{z} & \text { normal stresses } \\ \tau_{x y}, \tau_{y z}, \tau_{z x} & \text { shear stresses } \\ V_{f} & \\ {[K]} & \text { final domain volume } \\ & \\ & \\ V_{D} & \text { local stiffness matrix } \\ & \\ & \\ & \end{array}$




\section{CHAPTER 1}

\section{INTRODUCTION}

This introductory chapter will provide the reader with an overview of the project. The structure of the thesis will then be outlined to provide efficient navigation of the information and concepts discussed.

\subsection{Motivation for Thesis}

As the advancement in technology continues to grow with each passing decade, the implementation of new and profound research to that technology has become necessary. Companies are always searching for ways to design cost-effective items and structures, while at the same time maintaining its proper functionality. The growing field of topology optimization provides guidance for designers, and many methods to date have been successful in optimizing the mechanical performance of lightweight structures and components.

\subsection{Goals of the Thesis}

Previous work in the field of topology optimization has consisted of numerous methods for the identification of minimum weight topologies. The Prescribed Material Redistribution (PMR) scheme developed by Taggart and Dewhurst [1-5] has been implemented in Matlab for a two-dimensional analysis using four-node quadrilateral elements. This code identifies two-dimensional optimal topologies which can be extruded in the third direction. Codes have been developed to represent the extruded geometry in a 3-D STL file format. These models have been used to produce prototypes on 3-D printing machines. 
The thesis seeks to extend the Matlab script to include three-dimensional analysis. The primary task is the implementation of a three-dimensional Finite Element Analysis (FEA) solver in Matlab. Details of the 3-D formulation will be presented in this thesis. Due to the computationally intense nature of 3-D analysis, computationally efficient algorithms were implemented in order to minimize the run

time and memory requirements for each case. In addition to identifying techniques to improve computational efficiencies within the script, the 3-D code allows the user to impose half, quarter or one-eighth symmetry as appropriate for the particular structure being modeled. The final script is applied to identify optimal porous and composite material microstructures, depending on the given applied load conditions. For these cases, the symmetry functionality plays a large role in efficiently modeling the microstructure using appropriate unit cell models. Through post-processing mirroring of the unit cell results, an array of desired size can be generated.

\subsection{Structure of the Thesis}

The field of topology optimization is an emerging field in the design community and only recently is being incorporated in design software. This thesis seeks to provide an overview of the field and details for a 3-D implementation of the PMR method. The thesis contains six separate chapters which focus on different aspects of topology optimization.

Chapter 2 presents a literature review of the field of topology optimization. Major methods will be introduced, including the well-known SIMP and ESO algorithms, as well as the more recent PMR scheme. It will be shown that these methods have certain drawbacks, including common numerical problems such as 
checkerboarding and mesh dependency. Techniques to limit or eliminate these deficiencies will also be introduced.

Chapter 3 reviews the Finite Element Method, particularly as applied to threedimensional problems. A detailed formulation of the eight-node hexahedron element is developed. For these analyses, a simple 3-D array of cube-shaped elements is used to represent the design domain. A Cartesian coordinate system is employed with a systematic node numbering scheme. Details of the element formulation and basic stress and strain calculations are discussed, along with the main variables used in three-dimensions. The shape functions, Jacobian matrices, strain-displacement matrix, and stiffness matrices are then derived and shown in their proper equations. Terms that require the use of both local and global recognition are separated into sections and explained individually, such as the coordinate systems and stiffness matrices.

Chapter 4 discusses the Matlab code development. After a brief introduction to the Matlab programming language, the key contents of the three-dimensional PMR code will be discussed. These contents include an overview of the code formulation and notations used, along with a brief definition of key aspects of the code.

Chapter 5 contains the results of several test cases that were used to evaluate the code and to demonstrate its capabilities. The first main section explains the use of symmetry conditions in the script for certain cases. The cases demonstrate the codes ability to model various symmetry conditions and the corresponding computational advantages. A table of CPU times demonstrating the effectiveness of running symmetry functions is presented. The next set of test cases seek to identify optimal 
microstructures for porous and composite materials that are subjected to various threedimensional multi-axial stress states.

Chapter 6 concludes the thesis with a summary of the main findings of the research. Opportunities for future work associated with the project will be discussed. 


\section{CHAPTER 2}

\section{TOPOLOGY OPTIMIZATION}

This chapter aims to provide an overview of the field of topology optimization, including a literature review of recent contributions to this field. This review will include work from the eighteenth century where optimization techniques first originated, to the current twenty-first century where sophisticated optimization techniques are becoming commonplace in many industries. The numerous methods will be explained briefly, and the advantages and disadvantages will be discussed.

\subsection{Optimization History}

The existence of optimization methods can be traced as far back as the 1700's where prominent figures such as Newton, Cauchy, and Lagrange played a major role in developing important work related to the field of optimization [6]. Despite these theoretical contributions, development of practical optimization methods for use in industry did not begin until the early 1900's. In 1904, Michell [7] derived formulas

for minimum weight structures given different stress constraints and design domains. These Michell structures, as they were known, marked the beginning of the development of structural optimization methods. Later, in 1960, Schmit [8] introduced a new approach to structural optimization that would serve as a basis to many of the successful optimization methods used today. It was proposed that finite element structural analysis and non-linear mathematical programming could be combined to create optimal designs. The idea was considered revolutionary to many in relation to design methods at the time, therefore it was pursued extensively over the 
following decades. These developments were pursued in parallel with the rapid growth of high-speed computers and the incorporation of finite element analysis in computer-aided design.

\subsection{Topology Optimization}

By definition, the field of topology optimization is a mathematical approach that optimizes a material structure in order to satisfy a given set of design requirements. These requirements include the amount of material to be used in the final design, the geometry of the design domain, boundary conditions (BC's), and applied loads. Typically, the amount of available material is to be distributed into fully dense and void regions based on the results of the optimization within the design domain. Many iterations are performed to reach the optimum design in computeraided software programs. Frequently, optimized designs are used in the conceptual design process stage and then revised to further meet performance and manufacturability standards. The advantage to incorporating topology optimization into the design process leads to improved designs and the use of topology optimization in many industries is expanding. Even if the design does not yield a result that can be directly implemented, it does provide a benchmark optimal design that can be used to evaluate more feasible designs.

Topology optimization is also referred to as layout optimization or generalized shape optimization in many papers. Typically, two types of structures are considered: continuum and discrete structures. Continuum structures often refer to single parts or components, while discrete structures are typically larger structures such as trusses and bridges. 
Other forms of optimization analyses include size optimization and shape optimization. An example of each type is clearly illustrated in Figure 2.1. In shape optimization, the design parameters are considered and updated until the desired constraints are achieved. This includes fillets, chamfers, radiuses, material thickness, and many other design parameters. In size optimization, structures such as trusses and bridges are considered and analysis is done on the bars supporting the inner portions of the structure.

(a)
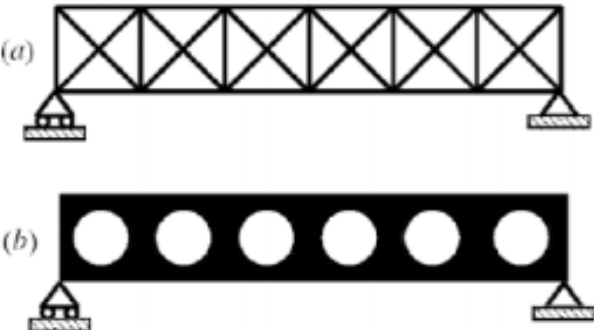

(c)
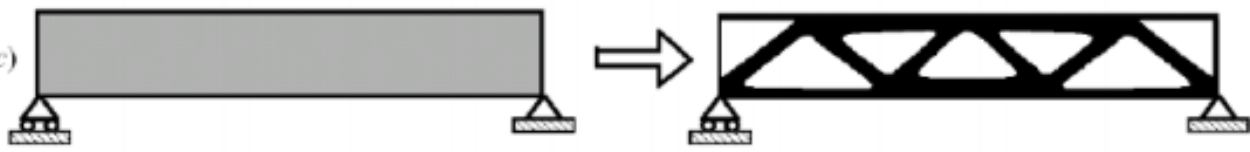

Figure 2.1: Examples of (a) Size Optimization, (b) Shape Optimization and (c) Topology Optimization [9]

\subsection{Homogenization Based Optimization}

The Homogenization Based Optimization (HBO) method was introduced in 1988 by Bendsøe and Kikuchi [9] and formed the basis for future topology optimization schemes. In the method, a small unit cell structure is designed and then homogenization is applied to determine the effective material properties of the individual cells. As an example, the method recognizes periodic microstructures and computes material properties of composites as demonstrated in Figure 2.2. 


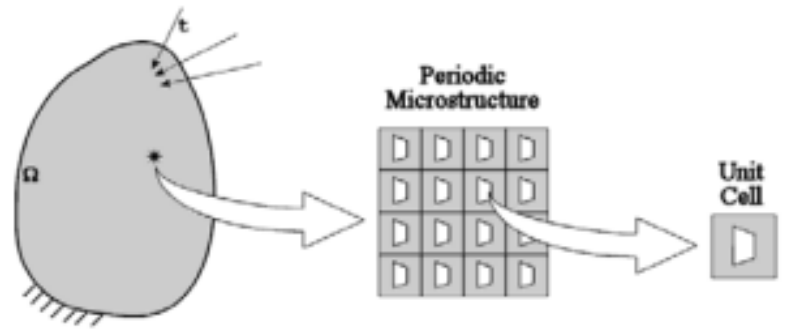

Figure 2.2: Illustration of composite material made of a periodic microstructure [10].

The unit cell to the far right is multiplied and shown in a $4 \times 4$ array in the center. The material stiffness in this case is portrayed as a function of the microstructure and density parameters [10].

$$
E=E^{0}(\rho, \theta, \mu \ldots)
$$

More recently, the HBO method has been replaced by a more effective method in which the unit cell consists of a partially dense, solid isotropic region. This method, called the Solid Isotropic Material with Penalization (SIMP) method, is described below.

\subsection{Solid Isotropic Material with Penalization}

The Solid Isotropic Material with Penalization (SIMP) method was originally introduced by Bendsøe [11] in 1989 and later developed independently by Rozvany et al. [12] in 1991. In this method, each element in the finite element mesh is considered to be partially dense. Through the course of several finite element iterations, the dense regions are redistributed in order to minimize the overall compliance (or maximize the overall stiffness) of the resulting structure. To inhibit the formation of regions with intermediate partial density, a penalization scheme is implemented and requires a heuristic penalization factor [13]. Due to SIMP's ability to successfully identify 
minimum weight topologies, it is widely used in the optimization field because of its computational efficiency and simplicity.

The primary drawback of the SIMP method is the sensitivity of the results to the penalty exponent, $n$, in the relation between local density, $\rho$, and Young's modulus

$$
E=\rho^{n} E^{0}, \quad 0 \leq \rho \leq 1
$$

The parameter, $n$, refers to the amount of penalization. For $n>1$, regions of intermediate density have reduced density and are therefore penalized. This parameter must be carefully selected to avoid results with an optimized structure containing regions of intermediate density. The desired result is distinct regions of fully dense material or regions with essentially zero density. In some cases, the user must explore the effect of the penalization parameter on the resulting optimized structure.

The flow chart of the SIMP method provided in Figure 2.3 gives a general overview of the procedure. The process begins with a uniform distribution of the densities in all of the elements in the design domain, based on the desired volume fraction specified by the user. A finite element analysis (FEA) is performed to determine the mechanical response for the current material distribution. A sensitivity analysis is applied to determine the relation between local element density and the overall compliance of the structure. These sensitivities are used to update the element densities for subsequent finite element iterations. To avoid numerical instabilities such as checkerboarding, a filtering technique is implemented before updating the element densities based on minimum compliance criteria. This entire process is 
repeated until the structure converges to fully dense or fully void regions. The converged structure represents the optimized design.

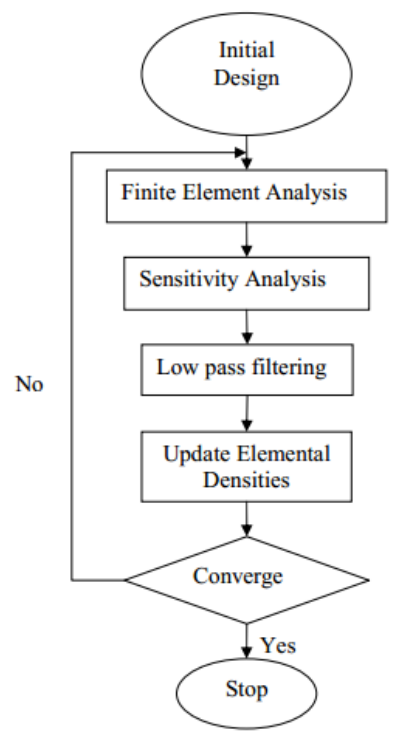

Figure 2.3 - SIMP flow chart [14]

In 2001, Ole Sigmund published an educational paper titled "A 99 line topology optimization code written in Matlab" [15] that demonstrates both the simplicity of the SIMP algorithm and the power of Matlab for use in topology optimization problems. Using a highly efficient 99 line Matlab code, Sigmund provides a complete compliance minimization scheme for use in identifying optimal topologies in statically loaded two-dimensional structures. The code developed in this study was initially based on Sigmund's efficient 2-D topology optimization code.

\subsection{ESO Algorithms}

Evolutionary Structural Optimization (ESO) algorithms are based on the simple concept that traditional structures are over-designed and therefore contain regions that are stressed well below the corresponding material strength. For that 
reason, improved designs can be achieved by removing unnecessary areas of dense material. In ESO type optimization methods, iterative finite element analyses are performed in which low stress regions are gradually removed from the structure. A few of these methods are described below.

\subsubsection{Evolutionary Structural Optimization}

In 1992, Xie and Steven [16] developed a novel method of topology optimization. The Evolutionary Structural Optimization (ESO) technique uses a finite element based iterative scheme for removing inefficient, low-stress material from a structure until an optimum design is reached. A common example of the ESO method is the design of structures exhibiting only tensile or compressive regions. For example, by identifying and removing the compressive regions in the structure, the expected theoretically optimal catenary structure can be attained as seen in Figure 2.4 [17].

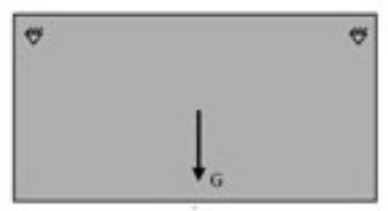

(a)

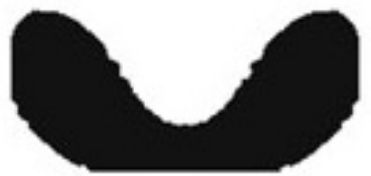

(b)

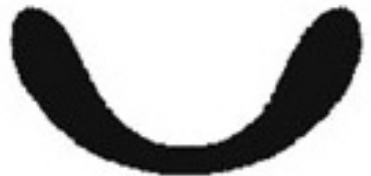

(c)

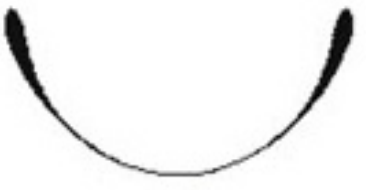

(d)

Figure 2.4: A catenary-type structure (a) Initial design (b)-(d) Evolution of optimal shape [17]

For the original method, once an element is removed from the structure, it could not be restored. This limitation led to the Bi-Directional Evolutionary Structural Optimization (BESO) method, which is discussed below. 


\subsubsection{Bi-Directional Evolutionary Structural Optimization}

In 1998, Querin et al. [18] proposed the Additive Evolutionary Structural Optimization (AESO) method that introduced the idea of material growth. When combined with the ESO-algorithm, a new method emerged. The Bi-Directional Evolutionary Structural Optimization (BESO) method allows for not only the removal of material to eliminate low-stress, but also the addition of material to regions of highstress. The number of elements removed or added is determined by the evolutionary ratio (ER) and the admission volume ratio (AR) [19]. To obtain the AR, the number of elements added are divided by the total number of elements in the initial design. Once the target volume of the structure is reached, the BESO algorithm is complete.

\subsection{Numerical Problems}

As with most numerical methods, there are deficiencies that may reduce the effectiveness of the method. Topology optimization is no exception and these deficiencies are well-documented in the literature. The most common types of numerical problems in topology optimization are checkerboarding and mesh dependence. Each of the sample problems discussed below are illustrated in Figure 2.5 . 


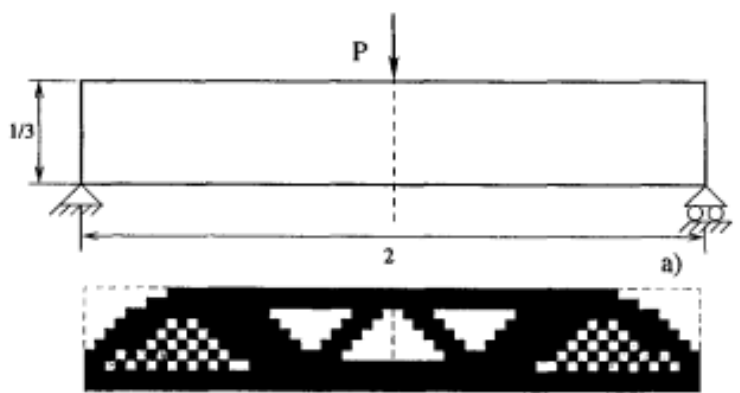

b)

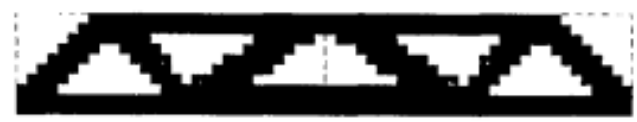

c)

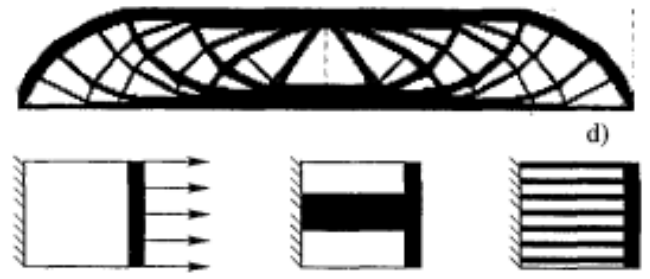

e)

Figure 2.5: (a) Design case, (b) Checkerboard example, (c) Solution for 600 element discretization, (d) Solution for 5400 element discretization, (e) Non-unique example [9]

\subsubsection{Checkerboarding}

Checkerboarding refers to the formation of regions in an optimal topology with alternating solid and void elements, hence the resemblance to a checkerboard. An example of a case undergoing checkerboard formulations can be seen clearly in Figure 2.5(b) for a simply-supported beam. It was originally believed that these checkerboards represented an optimum microstructure, but it was later discovered by Diaz and Sigmund [20] to be an artifact of numerical approximation associated with the finite element method. Because of this, the appearance of checkerboard patterns is interpreted as an unacceptable structural design. Prevention techniques have been 
developed for the elimination of checkerboarding. These techniques include patches, filtering, and higher-order finite elements.

The patch technique includes the formulation of an alternate finite element that diminishes the appearance of checkerboards in most cases. The technique allows the user to continue applying four-node quadrilateral elements, thus conserving CPU-time. The result of patching introduces a "super-element" with a higher number of nodes based on the density and displacement functions of the four surrounding elements. In topology optimization however, the technique does not remove the checkerboard effect entirely. Bendsøe [21] developed the patching process after he was inspired by similar problems in Stokes flow.

In 1994, Sigmund [22] introduced the filtering technique, similar to image filtering, where design sensitivities were altered and smoothed within each iteration. The design sensitivity of each element became dependent on the average weight of the element and its eight surrounding elements. In mathematical terms, the stiffness of a point $c$ depends on the density $x_{c}$ of all points in the surroundings, or neighborhood, of c. The application of this filter reduces the topology complexity of an optimized structure and ensures mesh-independency.

The use of higher-order finite elements for the displacement function can also eliminate the issue of checkerboarding. For the SIMP method, it is suggested that eight or nine-node quadrilateral elements be used to properly avoid checkerboards, along with a combination of an element wise constant discretization of density [23]. However, the penalization power $p$ needs to be small enough in order to obtain such results. A paper published by Diaz and Sigmund [20] in 1995 suggested that $p$ cannot 
be any larger than 2.29. Unlike the patching technique though, CPU-time is significantly increased under the higher-order finite element method because of the introduction of additional degrees of freedom. For this reason, although higher-order elements prevent checkerboarding, filtering techniques seem to be the preferred method for suppressing checkerboarding.

\subsubsection{Mesh Dependence}

Due to inherent approximations associated with finite element based methods, it is expected that the results of any finite element based topology optimization will be mesh dependent. As the finite element mesh is refined, the structure increases in detail and, in some cases, looks different all together. Figures 2.5(c) and 2.5(d) demonstrate an example of this where a model with 600 elements is compared to a 5400 element model. The 5400 element discretization model exhibits a highly detailed structure compared to the 600 element result.

Mesh-dependency results in a number of non-unique solutions for some cases based on the mesh size and discretization applied. Figure 2.5(e) provides an example of non-uniqueness where a structure undergoes uniaxial tension, but produces two drastically different optimized structures. One has a single thick bar in the center and the finer structure has several thin bars. Both models, however, are valid..

There are many techniques used for addressing mesh dependent problems such as relaxation, perimeter control and global and local gradient constraint. The meshindependency filter has proven to be the most successful technique to this date for three-dimensional cases. The mesh-independency filter is similar to the filtering 
technique introduced for checkerboarding, except here the filtered design sensitivities replace the real design sensitivities. Many users prefer the filtering technique primarily because it requires no extra constraints to the optimization problem and little to no extra CPU-time is needed.

\subsection{Prescribed Material Redistribution}

The Prescribed Material Redistribution (PMR) scheme was developed by Taggart et al. [1-5] as an alternative method to optimizing topologies. In PMR, the topology is identified through an iterative analysis where the relative material density $\rho(0<\rho \leq 1)$ is varied. Initially, the material mass is distributed uniformly throughout the desired design domain and results in a uniform, partially-dense material. All of the nodes are assigned an initial relative density

$$
\rho_{0}=\frac{V_{f}}{V_{D}}
$$

where $V_{f}$ is the final structural volume and $V_{D}$ is the final domain volume. The initial distribution and final cumulative distribution are defined, respectively, as

$$
\begin{gathered}
f_{0}(\rho)=\delta\left(\rho-\rho_{0}\right) \\
F_{0}(\rho)=H\left(\rho-\rho_{0}\right)
\end{gathered}
$$

where $f_{0}$ is the initial probability distribution function, $F_{0}$ is the cumulative distribution function, $\delta$ is the Dirac delta function and $H$ is the Heaviside step function. 
The final relative material density distribution consists of regions that are fully dense or nearly void, or $\rho=1$ and $\rho=\rho_{\min }$ (where $\rho_{\min } \ll 1$ ) respectively. The fully dense region is known as the optimal topology. The final probability distribution function $f_{F}$ and final cumulative distribution function $F_{F}$ are defined as:

$$
\begin{gathered}
f_{F}(\rho)=\left(1-\rho_{0}\right) \delta\left(\rho-\rho_{\text {min }}\right)+\rho_{0} \delta(\rho-1) \\
F_{F}(\rho)=\left(1-\rho_{0}\right) H\left(\rho-\rho_{\text {min }}\right)+\rho_{0} H(\rho-1)
\end{gathered}
$$

While the PMR method allows for various schemes for redistributing material, the use of Beta probability distribution functions has been demonstrated to be effective and efficient [1-5]. The gradual transition from the initial unimodal distribution to the final distribution of fully-dense or void regions through the use of the Beta function $\beta$ in Eq. (2.7.4).

$$
f_{\rho}=\beta(\rho, r, s)=\frac{\rho^{r-1}(1-\rho)^{s-1}}{\frac{\Gamma(\mathrm{r}) \Gamma(\mathrm{s})}{\Gamma(\mathrm{r}+\mathrm{s})}}
$$

where $\Gamma$ is the Gamma function and $r$ and $s$ are the adjustable parameters [4]. Then, the corresponding cumulative distribution function is imposed through the use of the incomplete Beta function $\beta_{\text {inc }}$ in Eq. (2.7.5).

$$
F_{\rho}=\beta_{i n c}(\rho, r, s)=\frac{1}{\beta(r, s)} \int_{0}^{\rho}\left(\rho^{\prime}\right)^{r-1}\left(1-\rho^{\prime}\right)^{s-1} d \rho^{\prime}
$$

Typical families of Beta and incomplete Beta distribution functions for the case $\rho_{0}=0.3$ are shown in Figures 2.6 and 2.7, respectively, where the evolution parameter, $t$, is initially zero and is monotonically increased to a final value of one. 
Appropriate functions $r(t)$ and $s(t)$ are specified [5] to provide a smooth transition from the initial to the final material density distribution.

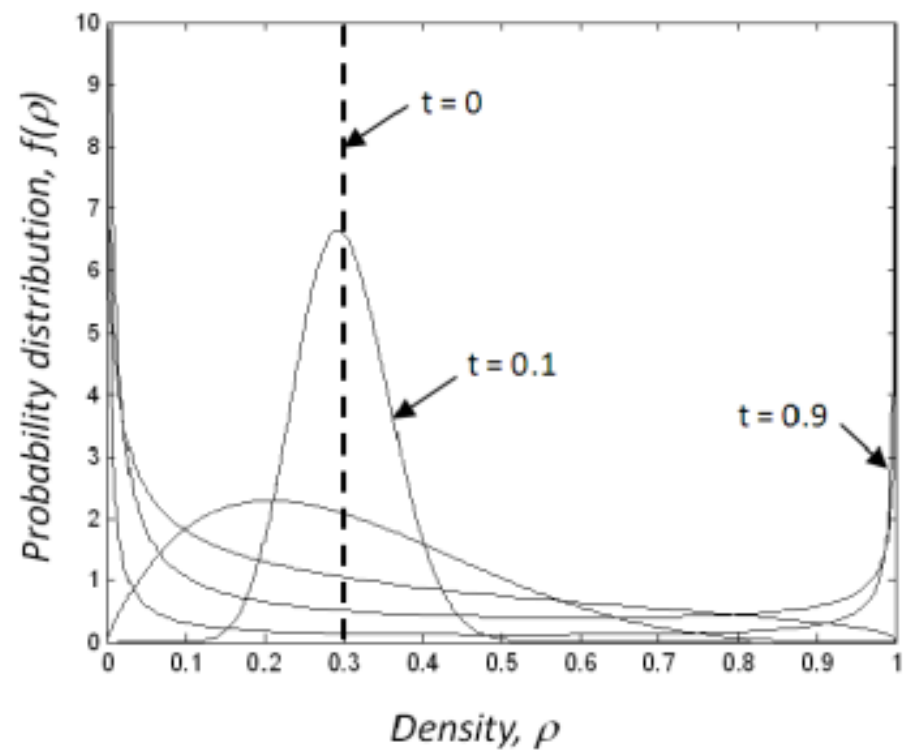

Figure 2.6: Transition from initial to final relative density distribution for the case $\rho_{0}=0.3[4]$

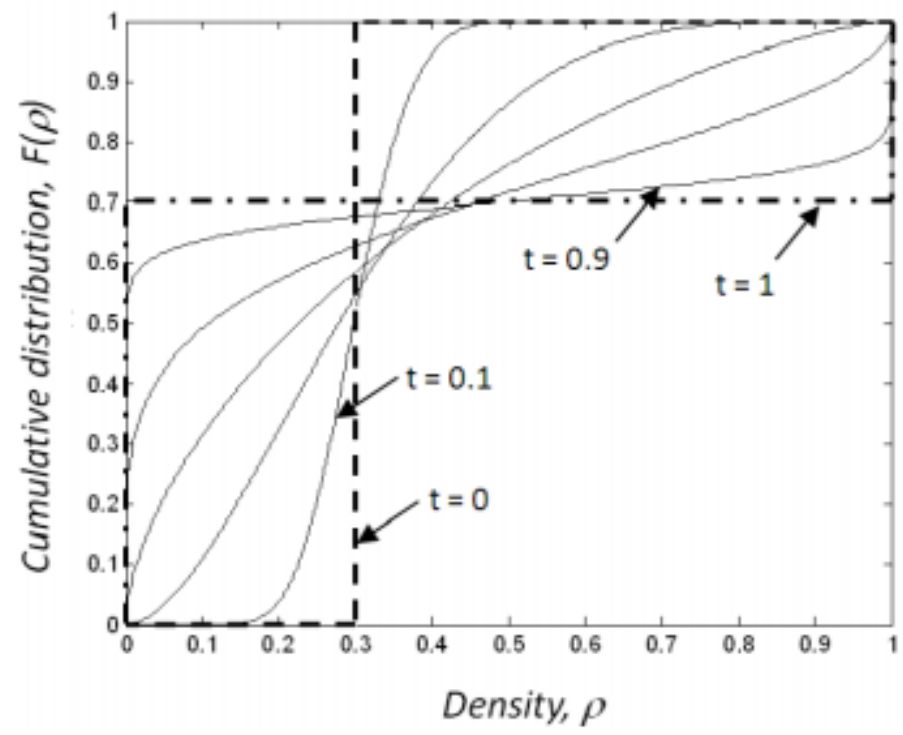

Figure 2.7: Transition from initial to final cumulative relative density distribution for the case $\rho_{0}=0.3$ [4] 
During each iteration, a detailed finite element analysis is performed. The nodal strain energy densities are computed and then sorted; the nodes with highest strain energy density are given highest relative density and the nodes with lowest strain energy density are given lowest relative density. The desired relative density distribution is then reached by assigning nodal densities based on sorted nodal strain energy densities.

The PMR scheme is able to successfully identify topologies in an optimized manner with high computational efficiency. Advantages include the enforcement of constant material volume as it is redistributed to areas of high strain energy density. It should be noted that the PMR scheme avoids the need for use of penalization factors and filtering methods that, as described above, are required in other optimization methods. 


\section{CHAPTER 3}

\section{FINITE ELEMENT ANALYSIS}

This chapter discusses the Finite Element Method (FEM) and its implementation in the three-dimensional PMR code. Element equations for threedimensional hexahedral elements are presented.

\subsection{Three-Dimensional Elements}

There are three basic groups of finite elements. These types include line elements for one-dimensional analysis, planar elements for two-dimensional analysis, and solid elements for three-dimensional analysis. Each group has their own subcategory of elements that pertain differently to specific applications.

The introduction of three-dimensional solid elements provides a more realistic approach to many applications in industry and research. Unlike two-dimensional elements, three-dimensional analyses avoid the need for plane stress or plane strain idealizations and can be used for general problems that are fully three-dimensional. The use of three-dimensional elements has been made feasible by the advancements in computer technology over the past few decades. There are a number of solid element types available for three-dimensional analysis. These include the tetrahedron, hexahedron and pentahedron elements. Each of the element types are illustrated in Figure 3.1. 

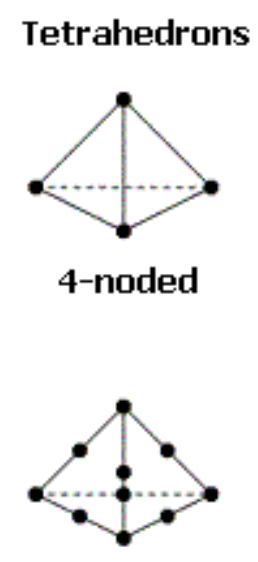

10-noded
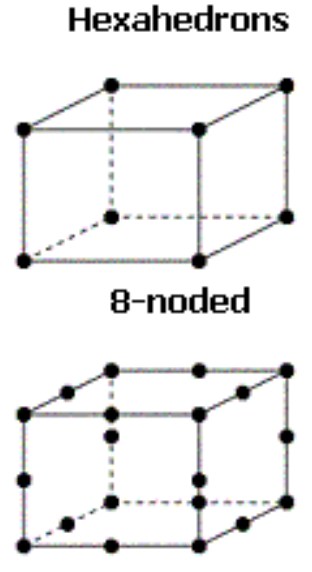

20-noded
Pentahedrons
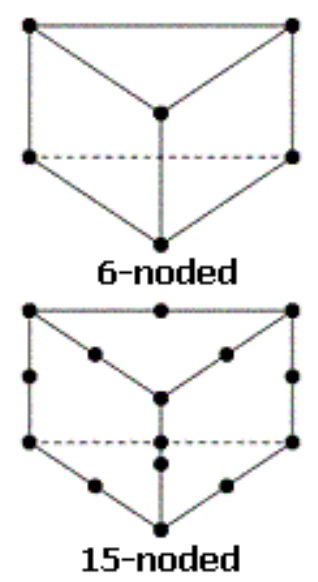

Figure 3.1: Three common element types in three-dimensional analysis [24]

The four-node tetrahedron, eight-node hexahedron, and six-node pentahedron are among the basic forms of each element type because nodes are located only at the corner points. The ten-node tetrahedron, twenty-node hexahedron, and fifteen-node pentahedron provide improved accuracy by including nodes at the midpoint between the corner point nodes. The additional nodes result in more degrees of freedom and higher-order displacement interpolation within each element. Although higher-order interpolation provides more realistic and accurate results, the run-time for each case is substantially increased.

The four-node tetrahedron, or "tet", is among the most popular and generalizes with the planar triangular element formulation in two dimensions. Tetrahedral elements provide the advantage of obtaining high quality meshes for complex geometries and are commonly used in adaptive mesh refinement schemes [25]. However, four node tetrahedral elements are known to provide low accuracy and hence, require very fine meshes for accurate results. The less common five-node 
pentahedron element, also known as a triangular prism or wedge, is used mostly for specialized applications such as the formation, propagation, and interrogation of cracks, as well as the interlaminar delaminations in composites due to impact loadings [25].

The element type selected for development in this thesis project is an eightnode hexahedron, which is a three-dimensional generalization of the two-dimensional quadrilateral element. Typically, hexahedral elements are taken to be rectangular prisms, in which case they are also known as a brick or cube element. These elements have eight nodes, six faces and twelve edges. Hexahedral elements are known to provide improved accuracy for three-dimensional problems as compared to four-node tetrahedral elements. An eight-node hexahedral element utilizes linear displacement interpolation functions. In contrast, the four-node tetrahedral element which also uses linear interpolation provides a state of constant strain within each element, resulting in high discretization errors. Many researchers prefer the use of hexahedral elements in non-linear problems because of severe locking problems associated with the use of tetrahedral elements. Since the hexahedral element formulation was selected for three-dimensional PMR method, the equations presented below are restricted to linear eight-node, hexahedral elements.

\subsection{Coordinate Systems}

As is common in most finite element formulations, the hexahedral element formulation involves consideration of two coordinate systems, a local coordinate system in which the element has a cube shape where typically each coordinate varies

from -1 to +1 and a global coordinate system which represents the global coordinates 
of the finite element mesh domain. Each coordinate system has its own node numbering system.

\subsubsection{Local Coordinate System}

The local coordinate system, or normalized coordinate system, is mainly used for numerical integration in computing the element stiffness matrix. The following corner numbering rule is applied to ensure a positive Jacobian determinant at every point in the element [25]. These rules are summarized as follows:

1. Choose one starting point, numbered node 1 , and one initial face which contains node 1 .

2. Number the other corner points 2, 3 and 4 in counterclockwise fashion (viewing from opposite face) on the initial face.

3. Number the opposite face the same way as the initial face, except 5, 6, 7 and 8 now.

The node numbering shown in Figure 3.2 below, followings these guidelines for the corner numbering rule and will be used in the element formulation 


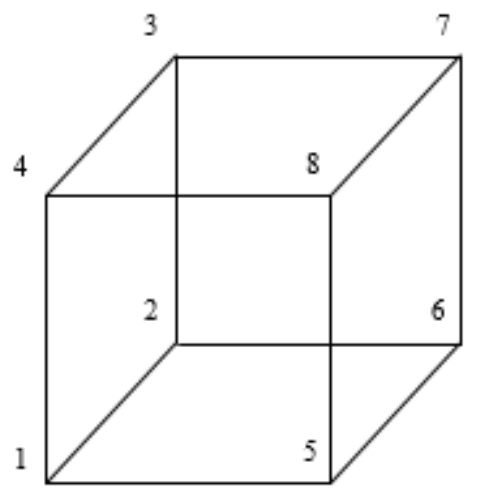

Figure 3.2: Local coordinate system for a hexahedral element with appropriate node numbering

The coordinate axes shown are represented by the symbols $s, t$, and $r$ with the origin of the axis located in the center of the element. Note that the formulation below follows the notation in Logan [26], with the exception that for simplicity in coding, $r$ will be used in place of Logan's $z$ ' coordinate. The coordinate locations of each of the eight nodes are shown in Table 3.1. Note that the cube coordinates vary from -0.5 to +0.5 such that the length of each edge is unity and the centroid of the element is located at the origin. Although this differs from the more commonly used variation from -1.0 to +1.0 , this difference is easily accounted for in the numerical integration of the element stiffness matrix, as will be shown below. 


\begin{tabular}{|c|c|c|c|}
\hline Node (i) & $\mathbf{s}_{\mathbf{i}}$ & $\mathbf{t}_{\mathbf{i}}$ & $\mathbf{r}_{\mathbf{i}}$ \\
\hline 1 & -0.5 & -0.5 & 0.5 \\
\hline 2 & -0.5 & -0.5 & -0.5 \\
\hline 3 & -0.5 & 0.5 & -0.5 \\
\hline 4 & -0.5 & 0.5 & 0.5 \\
\hline 5 & 0.5 & -0.5 & 0.5 \\
\hline 6 & 0.5 & -0.5 & -0.5 \\
\hline 7 & 0.5 & 0.5 & -0.5 \\
\hline 8 & 0.5 & 0.5 & 0.5 \\
\hline
\end{tabular}

Table 3.1: Node numbering and coordinate locations for local hexahedral element

\subsubsection{Global Coordinate System}

The global coordinate system, or reference coordinate system, describes the entire finite element domain being modeled. In general finite element formulations, the element hexahedral shape in global coordinates can take on an arbitrary shape. For this analysis, however, the element shape is taken to be a cube with unit length in all three directions. Figure 3.3 below displays an illustration of three eight-node hexahedral elements oriented along the $x$-axis and the node numbering scheme that has been implemented. 


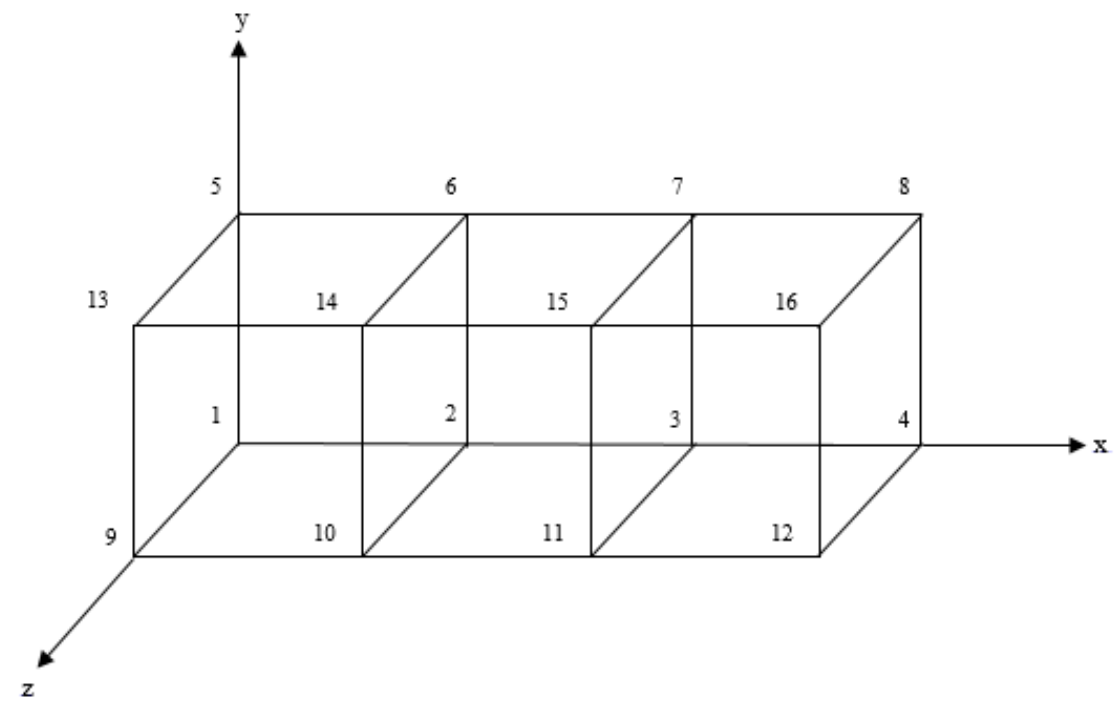

Figure 3.3: Global coordinate system for multiple hexahedral elements with appropriate nodal numbering.

The global coordinate axes symbols are taken to be $x, y$, and $z$ which are parallel to the local coordinates $s, t$, and $r$, respectively. The node numbering scheme starts with node 1 at the origin, followed by nodes 2,3 etc. along the $x$-axis. Then, node numbering continues at $(x, y, z)=(0,1,0)$ and continues for all nodes with $z=0$. This numbering procedure continues at $(x, y, z)=(0,0,1)$ and continues for all nodes with $z$ $=1$. This procedure continues for the remaining nodes. From the user point of view, specification of the number of nodes in the $x, y$ and $z$-directions (nelx, nely and nel z) fully defines the finite element mesh and the code automatically generates the node numbering scheme described above.

\subsection{Stresses and Strains}

A three-dimensional stress state consists of six different stress components. The normal stresses $\sigma_{x}, \sigma_{y}$ and $\sigma_{z}$ are perpendicular to the element faces. In general, these stresses change both the shape and volume of the material and are resisted by the 
body's elastic stiffnesses. In contrast, the shear stresses $\tau_{x y}, \tau_{y z}$ and $\tau_{z x}$ are parallel to the element faces. For isotropic materials, these stresses deform the material without changing the volume and are resisted by the body's shear modulus. For isotropic materials, the material stiffness is fully characterized by two elastic constants. In the formulation below, Young's modulus and Poisson's ratio are used to characterize the material stiffness. In Figure 3.4, the stresses are displayed on a typical three-dimensional element.

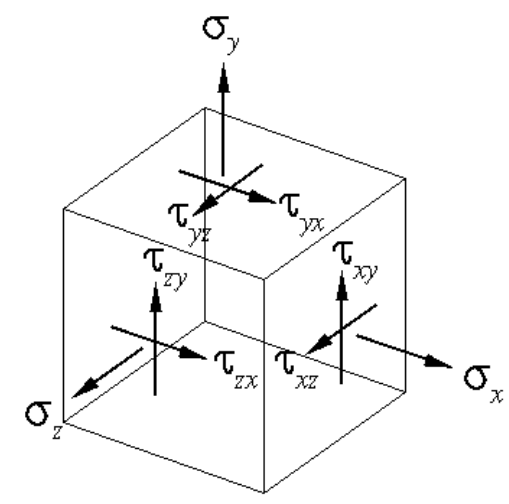

Figure 3.4: Three-dimensional element under a state of stress.

Similar to the stresses, there are six strain terms associated with a threedimensional strain state. These include the three normal strains $\varepsilon_{x}, \varepsilon_{y}$ and $\varepsilon_{z}$ and three engineering shear strains $\gamma_{x y}, \gamma_{y z}$ and $\gamma_{z x}$. The strains are proportional to deformation gradients according to the following strain-displacement relationships

$$
\begin{array}{ccc}
\varepsilon_{x}=\frac{\partial u}{\partial x} & \varepsilon_{y}=\frac{\partial v}{\partial y} & \varepsilon_{z}=\frac{\partial w}{\partial z} \\
\gamma_{x y}=\frac{\partial u}{\partial y}+\frac{\partial v}{\partial x} & \gamma_{y z}=\frac{\partial v}{\partial z}+\frac{\partial w}{\partial y} & \gamma_{z x}=\frac{\partial w}{\partial x}+\frac{\partial u}{\partial z}
\end{array}
$$


where $u, v$ and $w$ are the displacements in relation to the $x, y$ and $z$ coordinates, respectively. The element strains and element stresses can also be specified in column matrix form:

$$
\{\varepsilon\}=\left\{\begin{array}{c}
\epsilon_{x} \\
\varepsilon_{y} \\
\varepsilon_{z} \\
\gamma_{x y} \\
\gamma_{y z} \\
\gamma_{z x}
\end{array}\right\} \quad\{\sigma\}=\left\{\begin{array}{c}
\sigma_{x} \\
\sigma_{y} \\
\sigma_{z} \\
\tau_{x y} \\
\tau_{y z} \\
\tau_{z x}
\end{array}\right\}
$$

In matrix form, the stress-strain relationships can be written as

$$
\{\sigma\}=[C]\{\varepsilon\}
$$

where $[C]$ is the $6 \times 6$ elasticity matrix, also known as the constitutive matrix or material property matrix. For an isotropic material, whose stiffness values are the same in all directions, the elasticity matrix is defined in terms of Young's modulus E and Poisson's ratio $v$ in Eq. (3.3.4).

$$
[C]=\frac{E}{(1+v)(1-2 v)}\left[\begin{array}{cccccc}
1-v & v & v & 0 & 0 & 0 \\
v & 1-v & v & 0 & 0 & 0 \\
v & v & 1-v & 0 & 0 & 0 \\
0 & 0 & 0 & \frac{1-2 v}{2} & 0 & 0 \\
0 & 0 & 0 & 0 & \frac{1-2 v}{2} & 0 \\
0 & 0 & 0 & 0 & 0 & \frac{1-2 v}{2}
\end{array}\right]
$$

Young's modulus represents the ratio of axial stress to axial strain in uniaxial tension. Since a material under uniaxial tension must elongate in length, the value of $E$ is always greater than zero. Poisson's ratio represents the negative of the ratio of lateral strain to axial strain, and described as the lateral shrinkage in the material under 
uniaxial tension. Although the theoretical range of values for $v$ is from -1 to 0.5 , isotropic engineering materials typically exhibit a value between 0.25 and 0.35 [27].

\subsection{Shape Functions}

To accurately approximate the displacement field within the structure being analyzed, proper shape functions must be derived and implemented. The shape functions express the interpolation of the displacement function within the domain of

an element. For a linear displacement interpolation, the expression to define the shape function is

$$
N_{i}=\left(\frac{1}{2}+s s_{i}\right)\left(\frac{1}{2}+t t_{i}\right)\left(\frac{1}{2}+r r_{i}\right)
$$

where $s, t$ and $r$ are the local coordinates and $s_{i}, t_{i}$, and $r_{i}$ correspond to the coordinate locations of the $i^{\text {th }}$ node, again in local coordinates. Hence, for the eight node hexahedral element, the eight shape functions implemented are

$$
\begin{array}{ll}
N_{1}=\left(\frac{1}{2}-s\right)\left(\frac{1}{2}-t\right)\left(\frac{1}{2}+r\right) & N_{2}=\left(\frac{1}{2}-s\right)\left(\frac{1}{2}-t\right)\left(\frac{1}{2}-r\right) \\
N_{3}=\left(\frac{1}{2}-s\right)\left(\frac{1}{2}+t\right)\left(\frac{1}{2}-r\right) & N_{4}=\left(\frac{1}{2}-s\right)\left(\frac{1}{2}+t\right)\left(\frac{1}{2}+r\right) \\
N_{5}=\left(\frac{1}{2}+s\right)\left(\frac{1}{2}-t\right)\left(\frac{1}{2}+r\right) & N_{6}=\left(\frac{1}{2}+s\right)\left(\frac{1}{2} \pm t\right)\left(\frac{1}{2}-r\right) \\
N_{7}=\left(\frac{1}{2}+s\right)\left(\frac{1}{2}+t\right)\left(\frac{1}{2}-r\right) & N_{8}=\left(\frac{1}{2}+s\right)\left(\frac{1}{2}+t\right)\left(\frac{1}{2}+r\right)
\end{array}
$$

Each shape function reduces to a value of one at the node $i$ being referred to and zero at all other nodes. As is typical of finite element interpolation functions, the sum of the shape functions at any point in the element is a constant value of one. 


\subsection{Jacobian Matrix}

The partial derivation of shape functions with respect to the global coordinates $x, y$ and $z$ are required in the numerical integration for determining the element stiffness matrix. The derivatives of the shape functions are given as chain rule formulas, and shown in matrix form

$$
\left[\begin{array}{l}
\frac{\partial N_{i}}{\partial x} \\
\frac{\partial N_{i}}{\partial y} \\
\frac{\partial N_{i}}{\partial z}
\end{array}\right]=\left[\begin{array}{lll}
\frac{\partial s}{\partial x} & \frac{\partial t}{\partial x} & \frac{\partial r}{\partial x} \\
\frac{\partial s}{\partial y} & \frac{\partial t}{\partial y} & \frac{\partial r}{\partial y} \\
\frac{\partial s}{\partial z} & \frac{\partial t}{\partial z} & \frac{\partial r}{\partial z}
\end{array}\right]\left[\begin{array}{l}
\frac{\partial N_{i}}{\partial s} \\
\frac{\partial N_{i}}{\partial t} \\
\frac{\partial N_{i}}{\partial r}
\end{array}\right]
$$

where the $3 \times 3$ matrix is the inverse Jacobian $\left[J^{-1}\right.$. The Jacobian is expressed as

$$
[J]=\frac{\partial(x, y, z)}{\partial(s, t, r)}=\left[\begin{array}{lll}
\frac{\partial x}{\partial s} & \frac{\partial y}{\partial s} & \frac{\partial z}{\partial s} \\
\frac{\partial x}{\partial t} & \frac{\partial y}{\partial t} & \frac{\partial z}{\partial t} \\
\frac{\partial x}{\partial r} & \frac{\partial y}{\partial r} & \frac{\partial z}{\partial r}
\end{array}\right]
$$

and relates the local coordinates to the global coordinates. It can be regarded as a scale factor that multiples $d s d t d r$ to create the physical area increment $d x d y d z$. When computing the determinant of the Jacobian matrix, the notation $J$ is used.

\subsection{Strain-Displacement Matrix}

The displacement approximation in terms of shape functions is

$$
\{u\}=[N]\{d\}
$$

where $\{d\}$ refers to the nodal displacements in vector form. The relationship between strains and displacements is needed in FEA to further compute the element stiffness matrix and in computing the stress and strains from the nodal displacement results. 
The strain-displacement matrix $[B]$ is introduced and equated as a function of $s, t$ and $r$. Derivation of $[B]$ as a function of the shape functions $[N]$ is shown in Eqs. (3.6.2) and (3.6.3).

$$
\begin{gathered}
\{\varepsilon\}=[\partial]\{u\}=[\partial][N]\{d\}=[B]\{d\} \\
{[B]=[\partial][N]}
\end{gathered}
$$

Using Eq. (3.6.3), the strain-displacement matrix $[B]$ can be expanded and shown as the following $6 \times 24$ matrix:

$$
\begin{aligned}
& {[B]=\left[\begin{array}{cccccccccccc}
\frac{\partial N_{1}}{\partial s} & 0 & 0 & \frac{\partial N_{2}}{\partial s} & 0 & 0 & \frac{\partial N_{3}}{\partial s} & 0 & 0 & \frac{\partial N_{4}}{\partial s} & 0 & 0 \\
0 & \frac{\partial N_{1}}{\partial t} & 0 & 0 & \frac{\partial N_{2}}{\partial t} & 0 & 0 & \frac{\partial N_{3}}{\partial t} & 0 & 0 & \frac{\partial N_{4}}{\partial t} & 0 \\
0 & 0 & \frac{\partial N_{1}}{\partial r} & 0 & 0 & \frac{\partial N_{2}}{\partial r} & 0 & 0 & \frac{\partial N_{3}}{\partial r} & 0 & 0 & \frac{\partial N_{4}}{\partial r} \\
\frac{\partial N_{1}}{\partial t} & \frac{\partial N_{1}}{\partial s} & 0 & \frac{\partial N_{2}}{\partial t} & \frac{\partial N_{2}}{\partial s} & 0 & \frac{\partial N_{3}}{\partial t} & \frac{\partial N_{3}}{\partial s} & 0 & \frac{\partial N_{4}}{\partial t} & \frac{\partial N_{4}}{\partial s} & 0 \\
0 & \frac{\partial N_{1}}{\partial r} & \frac{\partial N_{1}}{\partial t} & 0 & \frac{\partial N_{2}}{\partial r} & \frac{\partial N_{2}}{\partial t} & 0 & \frac{\partial N_{3}}{\partial r} & \frac{\partial N_{3}}{\partial t} & 0 & \frac{\partial N_{4}}{\partial r} & \frac{\partial N_{4}}{\partial t} \\
\frac{\partial N_{1}}{\partial s} & 0 & \frac{\partial N_{1}}{\partial s} & \frac{\partial N_{2}}{\partial r} & 0 & \frac{\partial N_{2}}{\partial s} & \frac{\partial N_{3}}{\partial r} & 0 & \frac{\partial N_{3}}{\partial s} & \frac{\partial N_{4}}{\partial r} & 0 & \frac{\partial N_{4}}{\partial s}
\end{array}\right]} \\
& \left.\begin{array}{llllllllllll}
\frac{\partial N_{5}}{\partial s} & 0 & 0 & \frac{\partial N_{6}}{\partial s} & 0 & 0 & \frac{\partial N_{7}}{\partial s} & 0 & 0 & \frac{\partial N_{8}}{\partial s} & 0 & 0
\end{array}\right] \\
& \begin{array}{llllllllllll}
0 & \frac{\partial N_{5}}{\partial t} & 0 & 0 & \frac{\partial N_{6}}{\partial t} & 0 & 0 & \frac{\partial N_{7}}{\partial t} & 0 & 0 & \frac{\partial N_{8}}{\partial t} & 0
\end{array} \\
& \begin{array}{llllllllllll}
0 & 0 & \frac{\partial N_{5}}{\partial r} & 0 & 0 & \frac{\partial N_{6}}{\partial r} & 0 & 0 & \frac{\partial N_{7}}{\partial r} & 0 & 0 & \frac{\partial N_{8}}{\partial r}
\end{array} \\
& \begin{array}{llllllllllll}
\frac{\partial N_{5}}{\partial t} & \frac{\partial N_{5}}{\partial s} & 0 & \frac{\partial N_{6}}{\partial t} & \frac{\partial N_{6}}{\partial s} & 0 & \frac{\partial N_{7}}{\partial t} & \frac{\partial N_{7}}{\partial s} & 0 & \frac{\partial N_{8}}{\partial t} & \frac{\partial N_{8}}{\partial s} & 0
\end{array} \\
& \begin{array}{llllllllllll}
0 & \frac{\partial N_{5}}{\partial r} & \frac{\partial N_{5}}{\partial t} & 0 & \frac{\partial N_{6}}{\partial r} & \frac{\partial N_{6}}{\partial t} & 0 & \frac{\partial N_{7}}{\partial r} & \frac{\partial N_{7}}{\partial t} & 0 & \frac{\partial N_{8}}{\partial r} & \frac{\partial N_{8}}{\partial t}
\end{array} \\
& \begin{array}{llllllllllll}
\frac{\partial N_{5}}{\partial s} & 0 & \frac{\partial N_{5}}{\partial s} & \frac{\partial N_{6}}{\partial r} & 0 & \frac{\partial N_{6}}{\partial s} & \frac{\partial N_{7}}{\partial r} & 0 & \frac{\partial N_{7}}{\partial s} & \frac{\partial N_{8}}{\partial r} & 0 & \frac{\partial N_{8}}{\partial s}
\end{array}
\end{aligned}
$$

\subsection{Stiffness Matrix}

As a structure undergoes deformation through loading, the amount of resistance to that deformation is determined by the global stiffness matrix, which 
includes information related to both the geometry of the structure and the material behavior. In the finite element formulation, the global stiffness matrix is found by assembling the contribution of each of the element stiffness matrices.

\subsubsection{Local Stiffness Matrix}

The local stiffness matrix, or element stiffness matrix $[k]$ can best be generalized in mathematical terms as

$$
\{f\}=[k]\{d\}
$$

where $\{f\}$ refers to the nodal forces acting on a single element. By itself, the element stiffness matrix is singular, which means that it is non-invertible [27].

For an eight-node hexahedral element in three dimensions, the local stiffness matrix expands to a $24 \times 24$ matrix according to

$$
[k]=\int_{-0.5}^{0.5} \int_{-0.5}^{0.5} \int_{-0.5}^{0.5}[B]^{T}[C][B] J d s d t d r
$$

where the determinant of the Jacobian $J$ is required to transform from the global

coordinates to the local coordinates. Each of the eight node points in an element have three displacements, or degrees of freedom, in the $s, t$ and $r$ directions, resulting in a total of 24 degrees of freedom in the element. The element equations relate these degrees of freedom to the 24 nodal forces that act on the element.

\subsubsection{Global Stiffness Matrix}

The global stiffness matrix $[K]$ represents the entire structural domain and can be written as 


$$
\{F\}=[K]\{D\}
$$

where $\{F\}$ refers to the global forces and $\{D\}$ are the nodal displacements at the global level. According to the Maxwell Reciprocal Theorem, all stiffness matrixes are symmetric $[K]=[K]^{T}$. The global stiffness matrix is formed by expanding the element equations to system size and assembling them to form the global equations. These equations are modified to impose the displacement boundary conditions or constraint conditions. The resulting system of linear equations are then solved to determine the nodal displacements.

\subsubsection{Stress and Strain Calculation}

From the nodal displacements, Eqs. 3.3.4 and 3.6.2 are applied at the element level to compute the strains and stresses, respectively, within the element. Typically the stress and strain components are computed at the Gauss integration points, extrapolated to the nodal positions and averaged with the result from adjacent elements to provide an approximation of the nodal stresses and strain. The PMR topology optimization algorithm also requires calculation of the nodal strain energy, which can be computed directly from the nodal stress and strain results. 


\section{CHAPTER 4}

\section{THREE-DIMENSIONAL PMR CODE DEVELOPMENT}

This chapter discuss the structure of the Matlab script that has been developed to implement the three-dimensional PMR method. Following a short introduction of Matlab, the key sections of the script are explained in detail, providing reference to equations found in Chapter 3. The STL file generation is then introduced to explain how these files are created for use in viewing the optimized 3-D structures and for manufacture of components using 3-D printing technology.

\subsection{Matlab Introduction}

Matlab, short for "Matrix Laboratory", is a powerful computer programming language and interactive environment for code development. It is a commercial software produced and provided by The Mathworks, Inc. Matlab is widely used by scientists and engineers for matrix-based numerical computations and visualization. It has includes a wide range of built in mathematical functions and can produce a wide variety of graphical data visualizations. While Matlab was originally designed to provide matrix-based computations, it has evolved over the years into a powerful computational tool. Matlab is widely used as an instructional tool in universities for introductory and advanced courses in computer programming, mathematics, engineering and science. It is also widely used in industry and academia for research, analysis, engineering design and development. 


\subsection{Overview of Matlab Script}

The three-dimensional PMR Matlab script is divided into a number of functions. These functions are explained in detail in the following subsections, along with main terms translated and selected lines of code shown.

\subsubsection{Parameter Initialization}

Several parameters are to be specified by the user to define the particular optimization problem. Typically, the user will write a short script that defines the problem definition parameters. This script then calls the main function, pmr3D, which performs the PMR topology optimization. Through a built-in post-processing step, the optimized topology is converted to an STL file. STL is a standard graphic file format in which the geometry is defined by triangular domains. It can be used for viewing the 3-D geometry and manufacturing components using 3-D printing or other additive manufacturing technologies.

The user defined parameters include specification of the design domain, loading conditions, boundary conditions, number of PMR iterations (iter), and the desired final volume fraction (volfrac). The domain is taken to be a rectangular prism where the user specifies the number of elements nelx, nely and nely in the $x, y$ and $z$-directions, respectively. The finite element mesh that is used for the PMR analysis consists of cube-shaped elements where the length, width and height of each element is taken to be unity. Other parameters that the user must define are described below. 
For non-rectangular design domains, the user can define certain nodes to be assigned a very low density, in effect creating a void region. Alternatively, the user can define regions that are predefined to remain fully dense throughout the PMR iterations. Regions that are not taken to be void regions or fully dense are those to which the PMR algorithm is applied. Hence, these regions are considered to be the design domain. Three different region types need to be defined by the user according to values specified in Table 4.1.

\begin{tabular}{|c|c|c|}
\hline Region & Node Type & $\begin{array}{c}\text { Initial Nodal } \\
\text { Density }\end{array}$ \\
\hline numdesign & 1 & volfrac \\
\hline numvoid & 2 & 0.01 \\
\hline numdense & 3 & 1 \\
\hline
\end{tabular}

Table 4.1: Predefined regions with corresponding node type and node densities.

The final volume of the desired structure is specified through the volume fraction parameter, volfrac, which represents the ratio of the final desired volume to the volume of the region defined by the design nodes as specified by the number of design nodes, numdesign. As described in Chapter 2, the PMR algorithm initially assigns an intermediate density given by volfrac, to the design elements. At the end of the procedure, the fraction of fully dense design elements is volfrac, and the number of essentially void design elements is $(1-\mathrm{vol} f \mathrm{rac})$. The predefined void regions and fully dense regions are assigned nodal densities of 0.01 and 1 , respectively, the minimum and maximum density values. The value of 0.01 is used since a density value of zero would lead to zero stiffness, which in return would result in numerical difficulties for solving the finite element equations. 
The PMR algorithm is implemented by imposing a specified density distribution at each iteration through the use of the cumulative distribution functions described in Chapter 2. As detailed in Chapter 2, families of Beta distribution functions are used to impose a gradual transition from an initial state, where the design nodes have a uniform initial density, volfrac, to a final state where the design nodes are either nearly void or fully dense. The number of nodes that are fully dense is determined such that the ratio of the volume of fully dense domain to the volume associated with the design nodes is volfrac.

The loads and boundary conditions are specified by user defined array dbc and forces. The dbc array is an $N_{d b c} \times 6$ array in which columns 1 to 3 contain the $x, y$ and $z$ coordinates of nodes for which displacement boundary conditions are to be specified; columns 4 to 6 are either 0 or 1, with 0 specifying an unconstrained displacement boundary condition and 1 specifying a constrained displacement boundary condition. The number of rows in this array, $N_{d b c}$ is the number of nodes for which displacement boundaries are specified. All other nodes are taken to be unconstrained. The array, forces, is an $N_{\text {force }}$ x 6 array where columns 1 to 3 contain the $x, y$ and $z$ coordinates of nodes for which force conditions are to be specified and columns 4 to 6 are the $x, y$ and $z$ components of the force to be applied at that node. The number of rows in this array, $N_{\text {force }}$, is the number of nodes for which forces are specified. All other nodes are taken to have zero external forces applied.

Users can also specify other model parameters relating to imposing symmetry conditions. The array sym is a $1 \times 3$ array for which each value is 0 or 1 corresponding to the $x, y$ or $z$ coordinate axes. A value of 1 specifies that the plane 
normal to the corresponding axis represents a symmetry plane. The sym array is used in post-processing steps of STL file creation, where the parameters are used to generate appropriate mirroring of the final topology. Note that for cases where symmetry is desired, the user must define the appropriate displacement boundary conditions using the array $\mathrm{dbc}$, as described above. Finally, the user can impose constraint relations, where a given displacement of a given node, called the slave node, can be constrained to be equal to the corresponding displacement of another node, called the master node. These relations are defined through the array, constraint, where columns 1 to 3 are the $x, y$ and $z$ coordinates of the master node, columns 4 to 6 are the $x, y$ and $z$ coordinates of the slave node, and columns 7 to 9 are 0 or 1 , where the $x, y$ or $z$ component of the displacement of the slave node is forced to equal the corresponding displacement of the master node if the value is 1 .

The user parameters are sent to the pmr $3 \mathrm{~d}$ function using the command

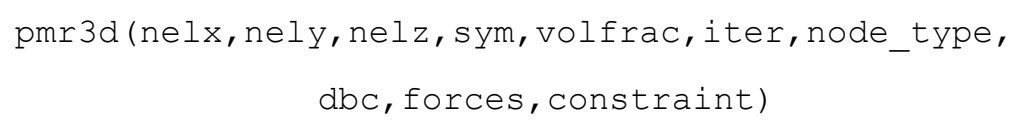

which performs iter iterations. For each iteration, the density distribution prescribed by the PMR algorithm is imposed and a finite element analysis is then performed to determine the resulting displacement, stress, strain and strain energy fields. The 3-D finite element solver is contained in the function, $\mathrm{FE}$, described below. It should be noted that the pmr $3 \mathrm{~d}$ function uses the user defined arrays $\mathrm{dbc}$, forces and constraint to define the arrays fixeddofs, $\mathrm{F}$ and $\mathrm{C}$ that are used as input to the function, $\mathrm{FE}$. 


\subsubsection{Finite Element Function}

The Finite Element $(\mathrm{FE})$ function performs a finite element analysis for the nodal density distribution corresponding to the current iteration. The call to the function $\mathrm{FE}$ is shown in Eq. (4.2.2) and the terms are defined in Table 4.2.

function [Unew, Ut] =FE (nelx, nely, nelz, fixeddofs, F, C, x, xvec)

\begin{tabular}{|c|c|}
\hline Matlab Code & Translation/Definition \\
\hline Unew & Strain energy at each node \\
\hline Ut & Total strain energy \\
\hline nelx & Number of elements in $x$-direction \\
\hline nely & Number of elements in y-direction \\
\hline nelz & Number of elements in z-direction \\
\hline fixeddofs & Fixed degrees of freedom \\
\hline F & Forces \\
\hline C & Constraints \\
\hline $\mathrm{x}$ & Density in 3-D matrix form \\
\hline xvec & Density values in vector form \\
\hline
\end{tabular}

Table 4.2: Matlab variable for the function FE

The function begins by initializing the key arrays as zeros or sparse. The command zeros assigns values of 0 to all terms in a matrix or array. The command sparse reduces memory storage by only storing non-zero values. The code computes the element stiffness matrices $(\mathrm{KE})$, the global stiffness matrix (K), the nodal displacement vector (D), the stresses and strains at the Gauss points (strsg, strng), the stresses and strains at the nodes (strsn, strnn), the strain energy at each node (Unew) and the total strain energy (Ut). The three-dimensional finite element equations developed in Chapter 3 are implemented in the function FE. In Table 4.3, 
key variables are defined, after which the corresponding equations or tables are shown.

\begin{tabular}{|c|c|c|}
\hline Matlab Code & Translation/Definition & Equation/Table \\
\hline $\mathrm{C} 0$ & Elasticity matrix & Eq. (3.3.4) \\
\hline $\mathrm{sn}$ & Node coordinates in $s$-direction & Table 3.1 \\
\hline $\mathrm{tn}$ & Node coordinates in $t$-direction & Table 3.1 \\
\hline $\mathrm{rn}$ & Node coordinates in $r$-direction & Table 3.1 \\
\hline $\mathrm{KE}$ & Element stiffness matrix & Eq. (3.7.2) \\
\hline $\mathrm{B}$ & Strain-displacement matrix & Eq. (3.6.4) \\
\hline $\mathrm{K}$ & Global stiffness matrix & Eq. (3.7.3) \\
\hline $\mathrm{N} 1 \mathrm{~s}$ & Shape function at node 1, derived by $s$ & Eq. (4.2.4) \\
\hline $\mathrm{N} 1 r$ & Shape function at node 1, derived by $t$ & Eq. (4.2.4) \\
\hline $\mathrm{N} 1 t$ & Shape function at node 1, derived by $r$ & Eq. (4.2.4) \\
\hline
\end{tabular}

Table 4.3: Matlab translations and corresponding equations/tables for FE function.

Due to the need for multiple computations of the B matrix, suppressed function Bmatrix was created to compute the B matrix as a function of $s, t$ and $r$.

$$
\text { function } B=B \text { matrix }(s, t, r)
$$

Within the function, the shape functions at any $s, t$ and $r$ location are defined when taking the derivatives of the interpolation functions with respect to each of the local coordinates $s, t$ and $r$.

$$
\begin{array}{lll}
N_{1 s}=-(0.5-t)(0.5+r) & N_{1 t}=-(0.5-s)(0.5+r) & N_{1 r}=+(0.5-s)(0.5-t) \\
N_{2 s}=-(0.5-t)(0.5-r) & N_{2 t}=-(0.5-s)(0.5-r) & N_{2 r}=-(0.5-s)(0.5-t) \\
N_{3 s}=-(0.5+t)(0.5-r) & N_{3 t}=+(0.5-s)(0.5-r) & N_{3 r}=-(0.5-s)(0.5+t) \\
N_{4 s}=-(0.5+t)(0.5+r) & N_{4 t}=+(0.5-s)(0.5+r) & N_{4 r}=+(0.5-s)(0.5+t) \\
& & \\
& & \\
N_{5 s}=+(0.5-t)(0.5+r) & N_{5 t}=-(0.5+s)(0.5+r) & N_{5 r}=+(0.5+s)(0.5-t) \\
N_{6 s}=+(0.5-t)(0.5-r) & N_{6 t}=-(0.5+s)(0.5-r) & N_{6 r}=-(0.5+s)(0.5-t) \\
N_{7 s}=+(0.5+t)(0.5-r) & N_{7 t}=+(0.5+s)(0.5-r) & N_{7 r}=-(0.5+s)(0.5+t) \\
N_{8 s}=+(0.5+t)(0.5+r) & N_{8 t}=+(0.5+s)(0.5+r) & N_{8 r}=+(0.5+s)(0.5+t)
\end{array}
$$

In developing the code, it was found that assembly of the global stiffness matrix $(\mathrm{K})$ requires a significant about of CPU-time. Typically, the global stiffness 
matrix assembly was more intensive than solving the global equations. In reviewing the literature, the stiffness matrix assembly method introduced by Alejandro OrtizBernardin [28] proved to be very effective in reducing CPU-time required.

In solving the global equations, imposition of displacement boundary conditions is simplified by defining which degrees of freedom are to be fixed and which are free. Following the code by Sigmund [15], the arrays alldofs and freedofs are defined as follows.

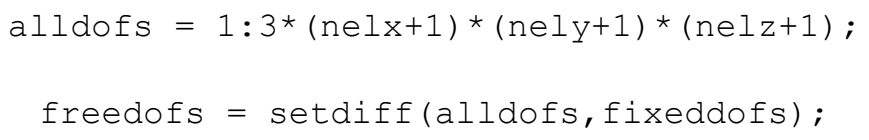

All of the degrees of freedom (alldofs) are calculated in the first equation of Eq. (4.2.5), which is based on three degrees of freedom for each node in the finite element mesh. Then, in the second equation of Eq. (4.2.5), the free degrees of freedom, freedofs, are determined by comparing alldofs to fixeddofs using the Matlab command setdiff. Note that the array fixeddofs is provided as input to the function $\mathrm{FE}$. With these definitions, solution of the global equations can be achieved with the command

$$
\mathrm{D}(\text { freedofs, }:)=\mathrm{K}(\text { freedofs, freedofs }) \backslash \mathrm{F}(\text { freedofs, }:) \text {; }
$$

After solving the global equations, the nodal stresses, strains and strain energies are computed using the finite element equations presented in Chapter 3. The array of nodal strain energies, Unew, are returned to the main $\mathrm{pmr} 3 \mathrm{D}$ function for use in updating the nodal densities for the next iteration. 


\subsubsection{Symmetry Function}

As mentioned above, the code provides the option to impose symmetry conditions to reduce the amount of CPU-time and memory required for more complex topology optimization problems. The combination of three-dimensional finite element analysis, along with a large number of nodes required for complex problems can be prohibitive in generating optimal designs. As a result, use of symmetry conditions when appropriate is desired.

A structure undergoing symmetrical loadings and boundary conditions can be analyzed within the PMR script. The symmetry displacement conditions are imposed through careful specification of the displacement boundary conditions, dbc. In such cases, the code creates appropriate mirroring of the optimal topology through the postprocessing creation of the STL geometry file. This is achieved using the function stl_sym_gen which performs the geometry mirroring. The call to this function is

$$
\begin{aligned}
& \text { function_[nx, ny, nz, node_def_new, el_def_new, dens_new] } . \\
& \text { =stl_sym_gen (nelx, nely, nelz, sym, node_def, el_def, dens) }
\end{aligned}
$$

In general, there are eight different cases of symmetry to consider under threedimensional analysis. The cases are acknowledged in the function as:

Case 1: no symmetry

Case 2: $x$-symmetry

Case 3: y-symmetry

Case 4: z-symmetry

Case 5: xy-symmetry

Case 6: yz-symmetry

Case 7: zX-symmetry

Case 8: full (xyz) symmetry 
To illustrate the procedure for mirroring the geometry, an excerpt of the stl_sym_gen function for Case 5 with xy-symmetry is given below.

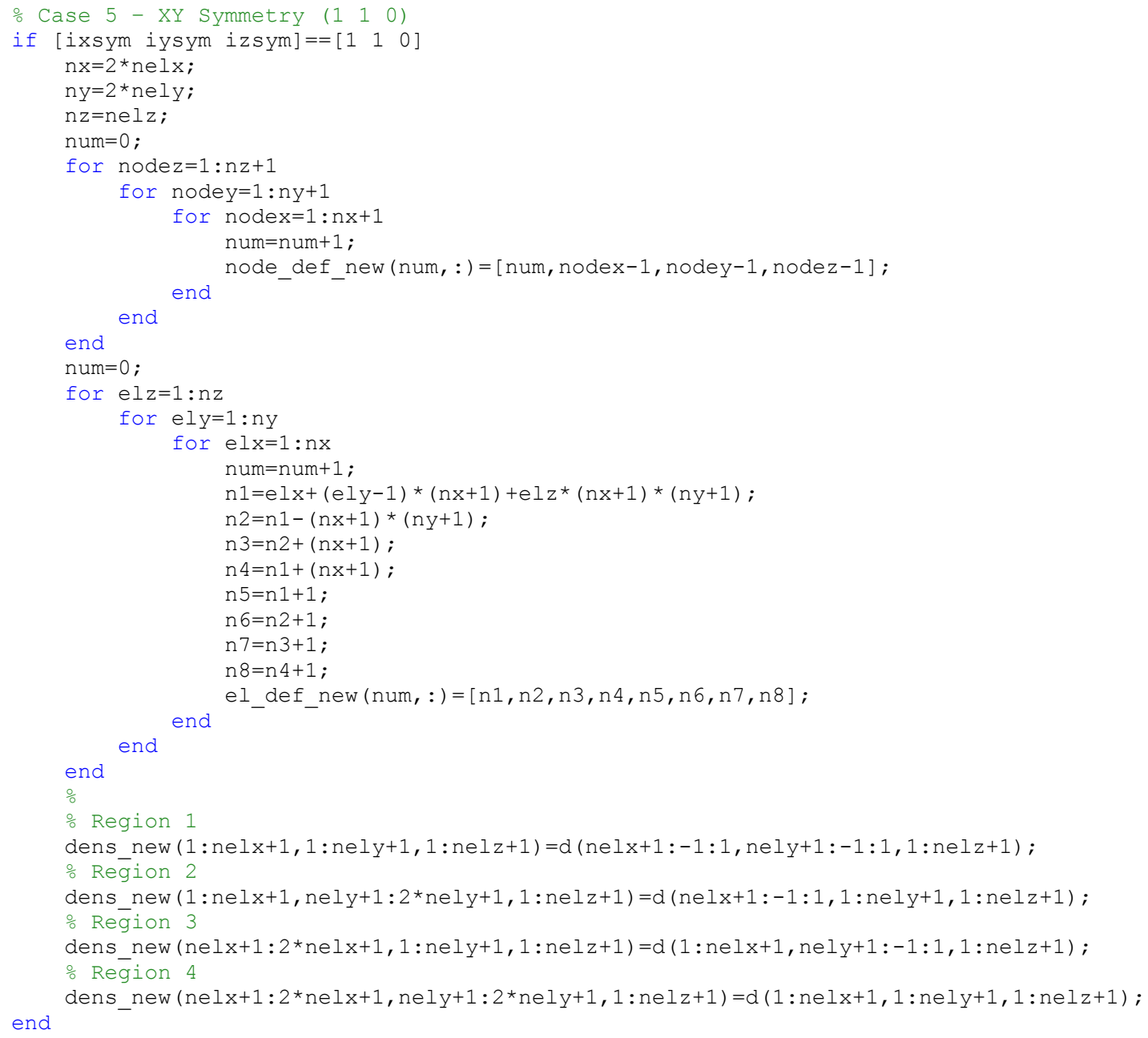

The specified plane of symmetry is determined by assigning a value to ixsym, iysym and izsym, which impose symmetry along the $x, y$ and $z$ plane, respectively. A value of 1 is assigned to denote the active symmetry while a value of 0 is inactive. The dens_new term takes the optimized nodal density distribution and mirrors it about the appropriate symmetry planes. The new density distribution, dens_new, is then used in the STL generation function, described below. 


\subsection{STL File Generation}

After the finite element results have been converged, the final bimodal material distribution data is converted into contour surfaces of constant density. The contours are then saved in STL format, which was originally implemented for use in the stereolithography prototyping process but has also been identified as Standard Tessellation Language. The STL file can be transmitted and viewed in standard CAD viewer software. A free program called 3D Myriad Reader [29] was used throughout the thesis project to view results of the case studies. The STL file can also be used as direct input for producing prototypes with a rapid manufacturing 3-D printer or any additive manufacturing process. 


\section{CHAPTER 5}

\section{ANALYSIS OF RESULTS}

This chapter presents the results of several test cases that were developed to validate the code and to demonstrate its capabilities. The first set of test cases demonstrate the capability to model symmetry conditions by modeling the same optimization problem with various symmetry conditions. The test case selected is a

simply-supported, centrally-loaded structure for which the minimum weight optimized solution is well-known. The series of test cases focuses on optimizing the microstructure of either porous or two-phase composite materials. Several different cases for various multi-axial stress states are considered. Microstructures are then developed through symmetry of unit cells and replication of unit cell results as a postprocessing operation.

\subsection{Symmetry Conditions}

To demonstrate the effectiveness of each case, the same example is run for the first seven symmetry cases described below. This test case is based on a classical Michell-arch structure. A.G.M. Michell [7] established the theoretical foundation of minimum-weight structures in 1904 and showed that minimum weight structures contain members subjected to uniaxial tension and compression, in a curved network corresponding to directions of principal strain. The example considered in the evaluation of the PMR scheme is for the case of a center load applied mid-span between two pinned supports. The PMR design domain is extended beyond the support points such that the optimized structure lies fully within the specified domain. 
The theoretical optimized Michell-arch structure for this loading (see Figure 5.1) consists of radial members to the central load point on an arch structure to the support points.

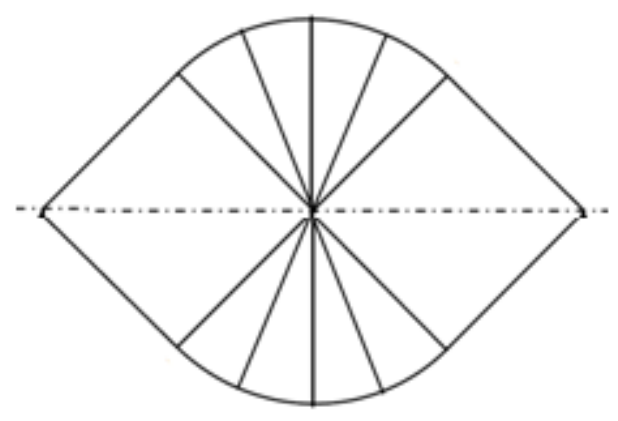

Figure 5.1: Theoretical optimal Michell-arch structure.

Seven test cases were developed using various combinations of symmetry planes as listed in Table 5.1. It can be seen that for all cases, the PMR method gives a reasonable representation of the theoretical optimal solution. The total CPU-time for each case has been recorded in minutes and the main parameters are specified in Table 5.1 below.

\begin{tabular}{|c|c|c|c|c|c|c|c|c|}
\hline Case & volfrac & nelx & nely & nelz & $\begin{array}{c}\text { Total } \\
\text { DOF's }\end{array}$ & iter & $\begin{array}{c}\text { CPU } \\
(\mathbf{m i n})\end{array}$ & $\begin{array}{c}\text { Efficiency } \\
(\%)\end{array}$ \\
\hline 1 & 0.16 & 48 & 42 & 10 & 60480 & 100 & 76.4 & 0 \\
\hline 2 & 0.16 & 24 & 42 & 10 & 30240 & 100 & 32.6 & 57 \\
\hline 3 & 0.16 & 48 & 5 & 42 & 30240 & 100 & 30.2 & 60 \\
\hline 4 & 0.16 & 48 & 42 & 5 & 30240 & 100 & 29.9 & 61 \\
\hline 5 & 0.16 & 24 & 5 & 42 & 15120 & 100 & 15.2 & 80 \\
\hline 6 & 0.16 & 24 & 42 & 5 & 15120 & 100 & 15 & 80 \\
\hline $6 \mathrm{~B}$ & 0.06 & 40 & 70 & 8 & 67200 & 100 & 86.2 & N/A \\
\hline 7 & 0.16 & 42 & 5 & 24 & 15120 & 100 & 14.6 & 81 \\
\hline
\end{tabular}

Table 5.1: Total CPU-time results for similar Michell-arch example using first seven symmetry cases 
The efficiency column refers to the percentage of time eliminated in comparison with Case 1 exhibiting no symmetry. The data reveals that the simplest form of symmetry $(x, y$, or $z)$ results in a total time reduced to more than half; with other symmetries reduced to more than three-quarters. It is important to note that these times will vary depending on the computer being used; the efficiency values, however, should remain approximately the same. For Case 6B, the number of elements were increased, which, as expected, resulted in an increased CPU-time. In this case, a smaller volume fraction, 0.06, was used, resulting in the structure shown in Figure 5.2. The predicted topology is consistent with the theoretical Michell-arch structure. Structures from the remaining seven cases are shown in Appendix A.

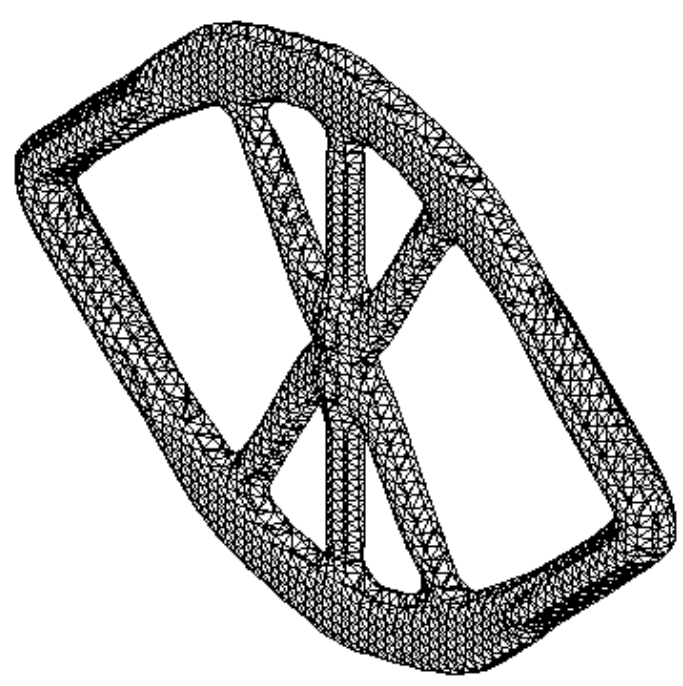

Figure 5.2: Michell-arch result using xy-symmetry for Case $6 B$

The Michell-arch case is not appropriate for validating the full symmetry feature due to its geometrical orientation and loading conditions. Therefore, a separate 
unique case was considered in order to validate the effectiveness of Case 8 . The loading conditions for this case are shown in Figure 5.3, where the outlined red region represents the section being mirrored amongst the other seven regions.

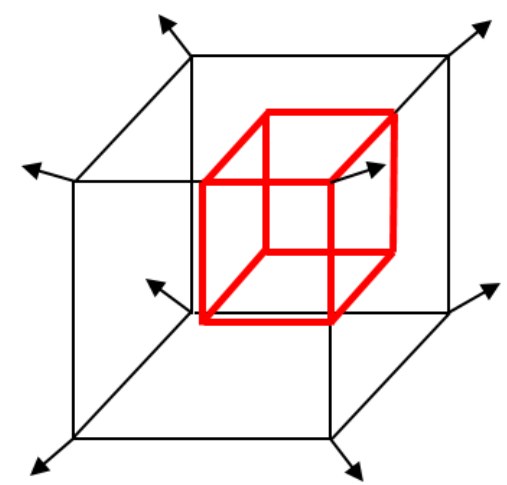

Figure 5.3: Initial conditions for Case 8 with full symmetry

The example was run for three different conditions as shown in Table 5.2. In Case 8A, no symmetry was used with twenty elements in each of the three directions. For Case $8 \mathrm{~B}$ and $8 \mathrm{C}$ however, full symmetry was used with ten elements and twenty elements, respectively, in each of the three directions. Results for Cases, $8 \mathrm{~A}, 8 \mathrm{~B}$ and 8C are displayed in Figure 5.4 below. It can be seen that through the use of symmetry, e.g. Case $8 \mathrm{~A}$ vs. $8 \mathrm{~B}$, dramatic reductions in CPU-time can be achieved. Alternatively, use of symmetry can be used to provide more precise results, e.g. Case $8 \mathrm{~A}$ vs. $8 \mathrm{C}$, with comparable CPU-times.

\begin{tabular}{|c|c|c|c|c|c|c|c|}
\hline Case & volfrac & nelx & nely & nelz & $\begin{array}{c}\text { Total } \\
\text { DOF's }^{\prime}\end{array}$ & iter & $\begin{array}{c}\text { CPU } \\
\text { (min) }\end{array}$ \\
\hline 8A & 0.1 & 20 & 20 & 20 & 24000 & 100 & 32.4 \\
\hline 8B & 0.1 & 10 & 10 & 10 & 3000 & 100 & 2.9 \\
\hline 8C & 0.1 & 20 & 20 & 20 & 24000 & 100 & 34.6 \\
\hline
\end{tabular}

Table 5.2: Total CPU-time results for similar unique example using no symmetry (Case $8 A$ ) and full symmetry (Case $8 B$ and $8 C$ ) 

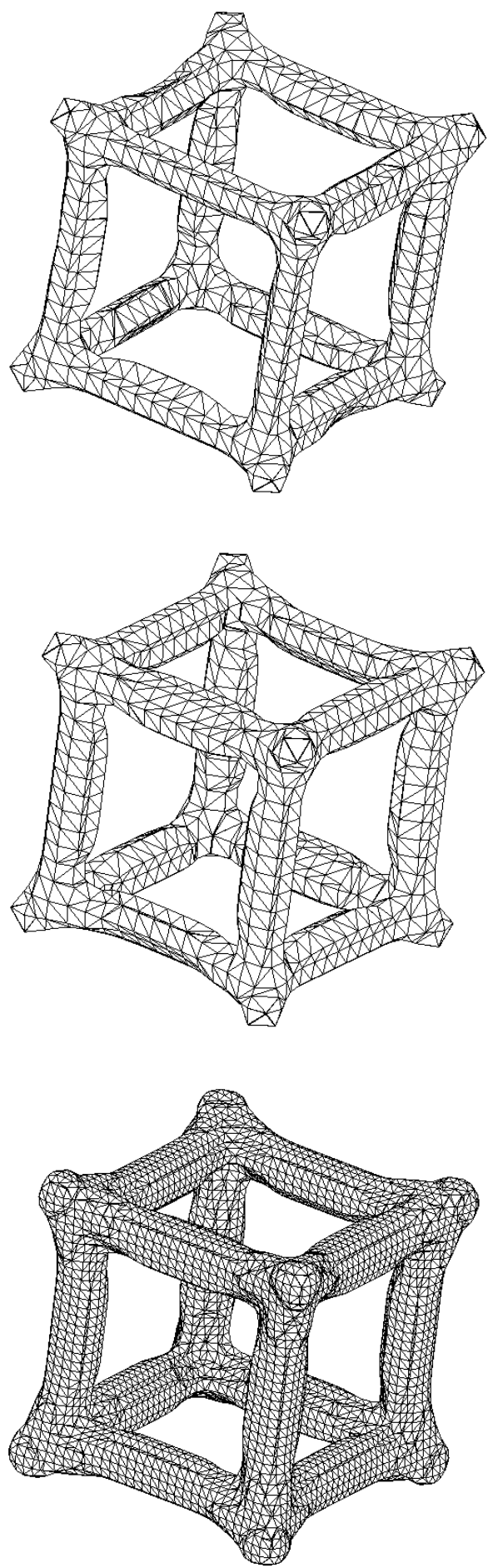

Figure 5.4: Full symmetry test cases results: Case 8 A (top), Case $8 B$ (middle) and $8 C$ (bottom) 


\subsection{Microstructures}

This section will focus on developing microstructures using one-eighth unit cell models, which are mirrored to the other seven regions using symmetry conditions. In Figure 5.5 below, the initial region selected for the unit model is designated by the dashed red outline.

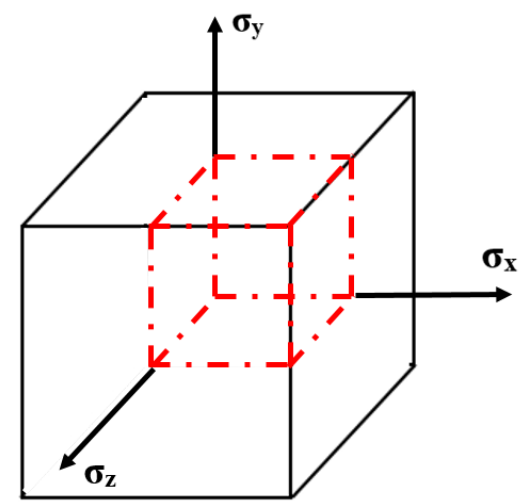

Figure 5.5: Outline of unit cell model region

In order to accurately develop a final microstructure, proper constraints must be applied to the faces of the unit cell. On the interior faces, displacements normal to the plane are zero and on the exterior faces, displacements normal to the plane are uniform. In Figure 5.6, these constraints are shown with the interior faces color-coded based on the applied constraint. The green face applies zero displacement in the $\mathrm{x}-$ direction, the red face for the y-direction, and the blue face for the z-direction. 


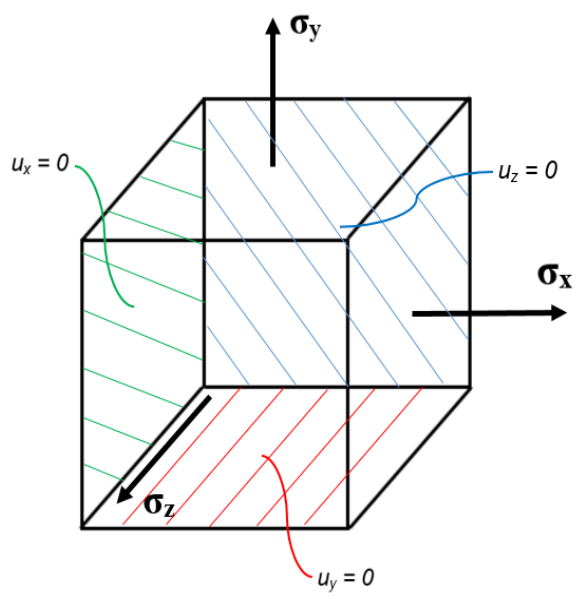

Figure 5.6: Unit cell model with constraints shown

\subsubsection{Porous Microstructures}

A number of cases were considered for various stress states. Each case utilized a volume fraction of 0.2 and the number of iterations was taken to be 100 . To model a porous material, the fully dense domain was assigned a Young's modulus of 1.0 and the void region was assigned a modulus of 0.01 . As specified in Table 5.3, various multi-axial stresses were then applied. Table 5.3 shows the stress states for each case and the corresponding microstructures in Figure 5.7.

\begin{tabular}{|c|c|c|c|}
\hline Figure 5.7( ) & $\boldsymbol{\sigma}_{\mathbf{x}}$ & $\boldsymbol{\sigma}_{\mathbf{y}}$ & $\boldsymbol{\sigma}_{\mathbf{z}}$ \\
\hline $\mathrm{a}$ & 1 & 0 & 0 \\
\hline $\mathrm{b}$ & 1 & 1 & 0 \\
\hline $\mathrm{c}$ & 1 & -1 & -1 \\
\hline $\mathrm{d}$ & 1 & 0.5 & 0.5 \\
\hline $\mathrm{e}$ & 1 & 0.75 & 0.75 \\
\hline $\mathrm{f}$ & 1 & 1.25 & 1.25 \\
\hline $\mathrm{g}$ & 1 & 1.5 & 1.5 \\
\hline $\mathrm{h}$ & 1 & 0.5 & 1 \\
\hline
\end{tabular}

Table 5.3: Stress values for each porous microstructure figure 
(a)
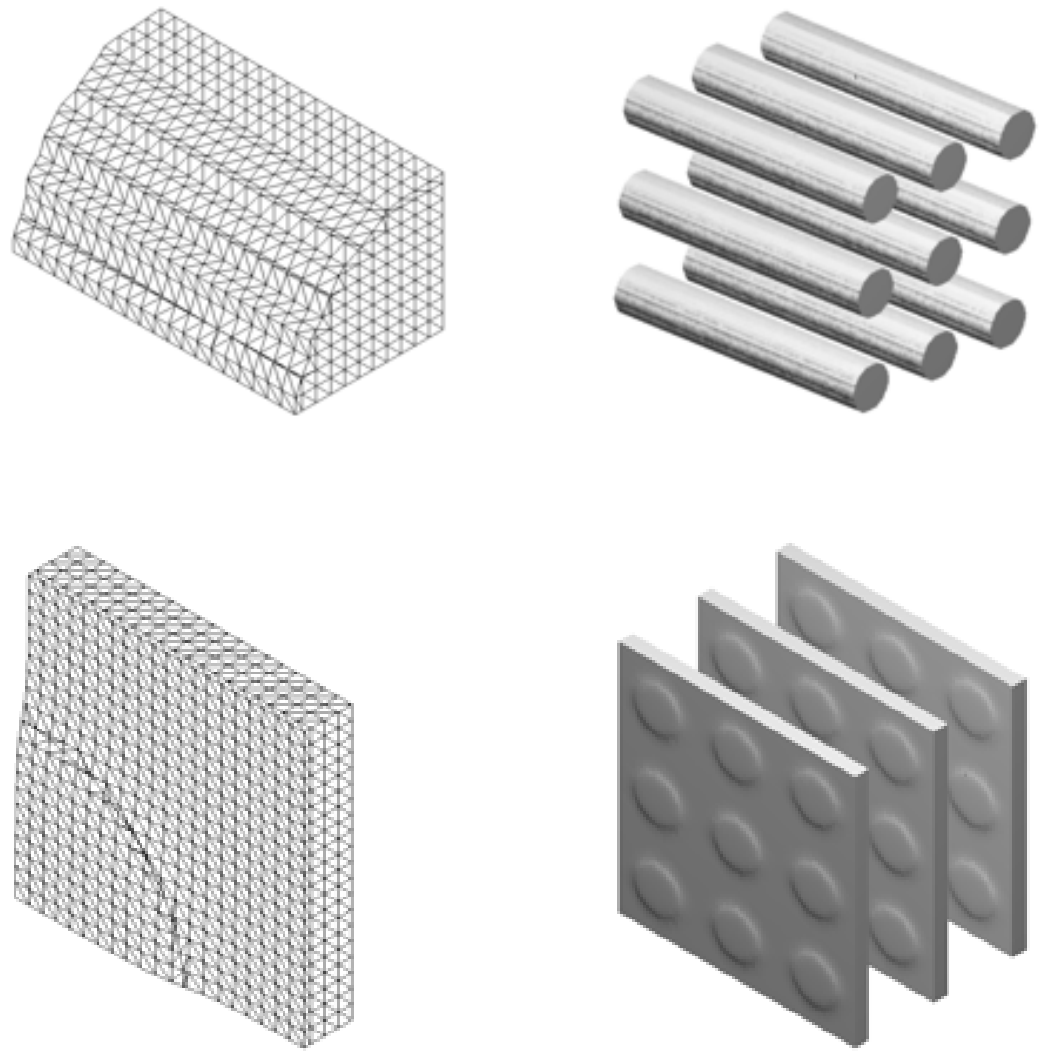

(b)

(c)
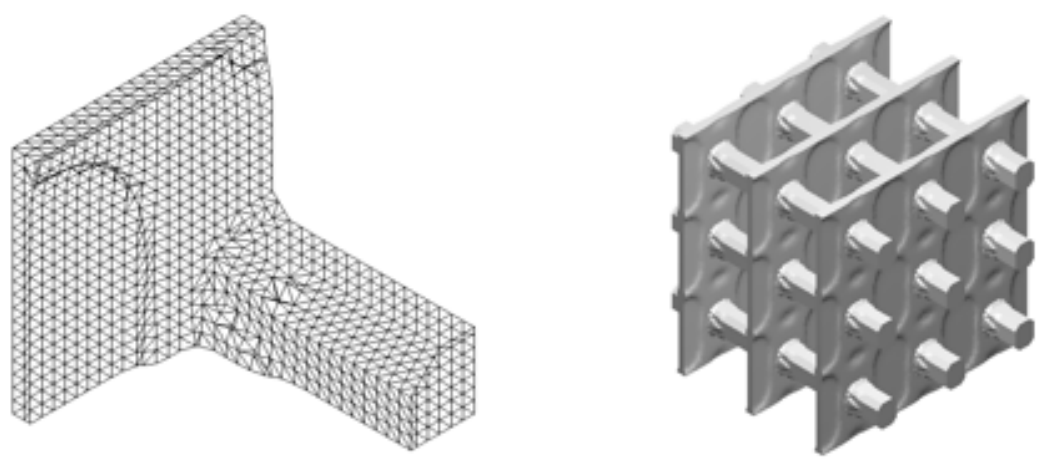

(d)
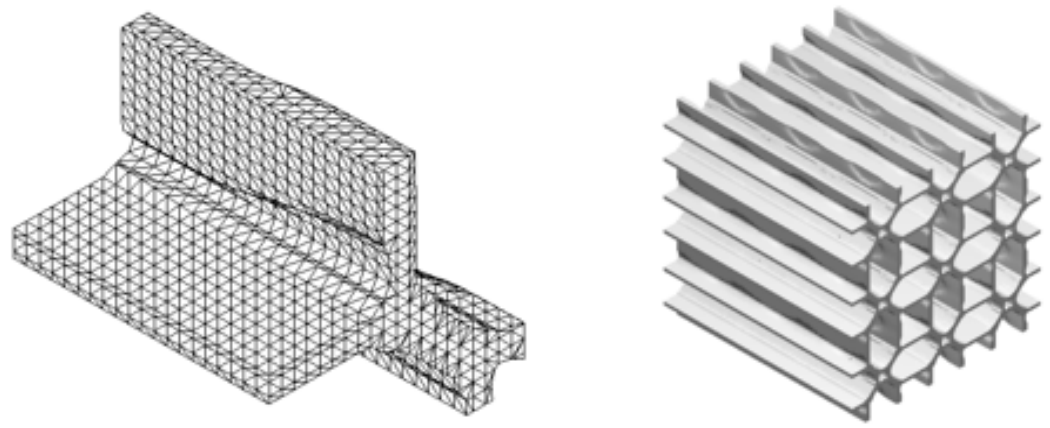
(e)
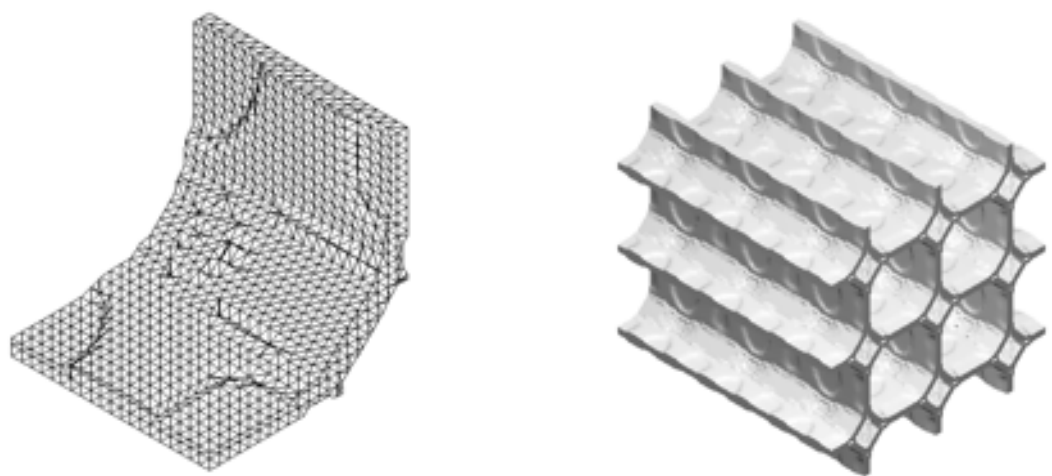

(f)
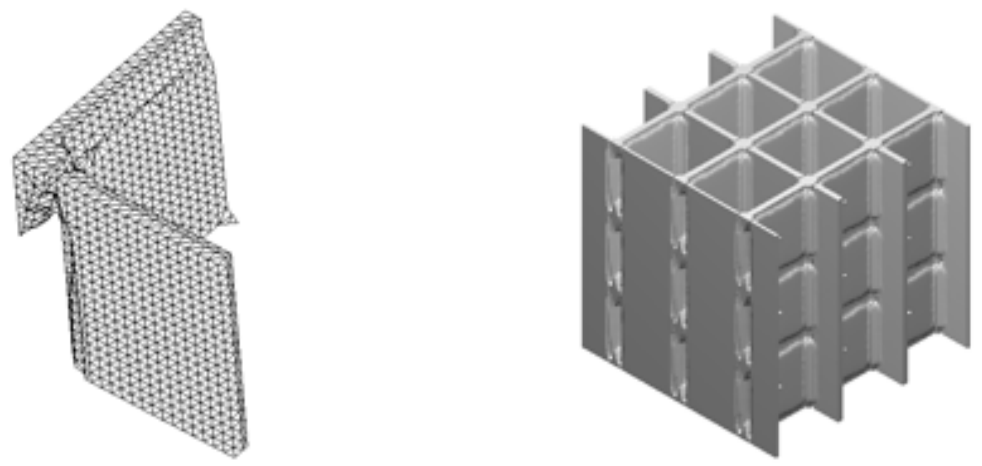

(g)
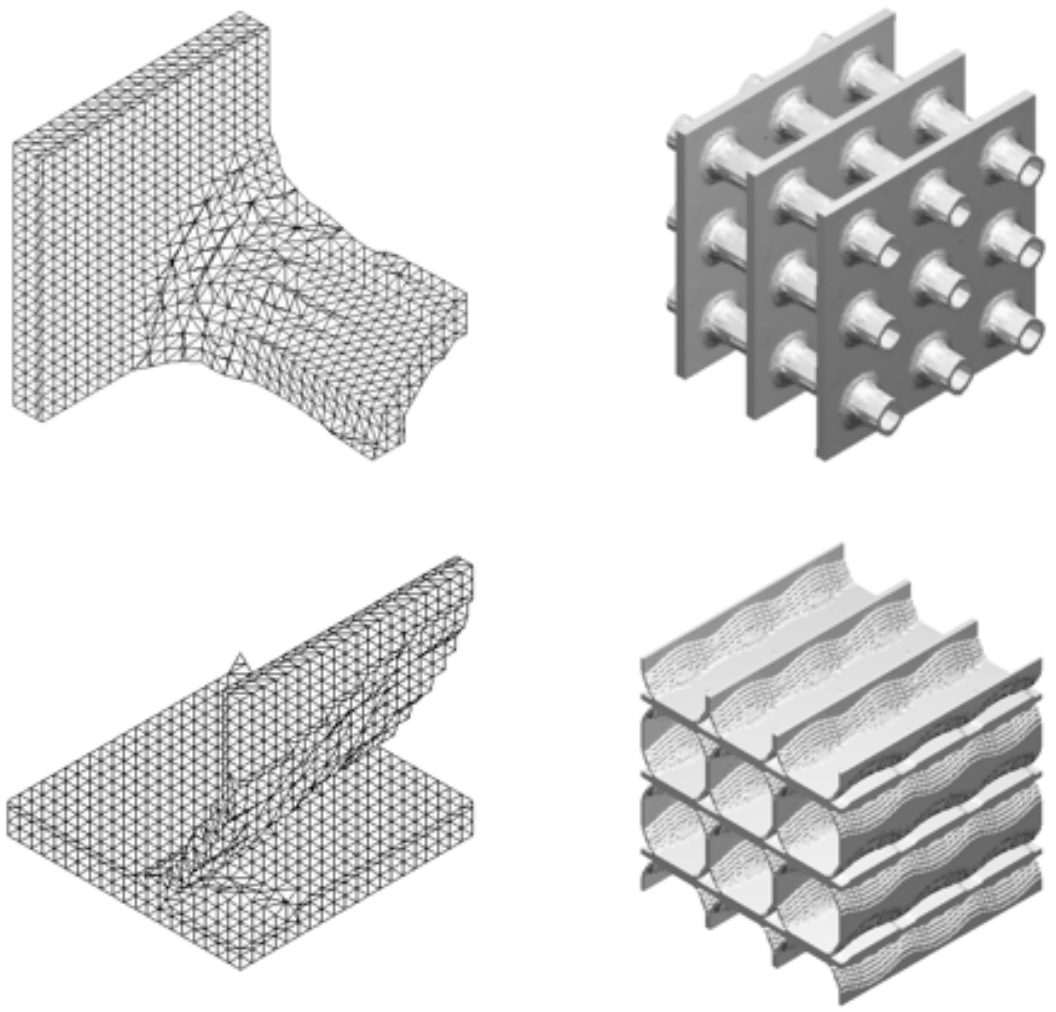

(h)

Figure 5.7: Microstructures made of porous materials; (a) through (h) cases correspond to conditions in Table 5.3 
For each case, the image on the left refers to a quarter of the material's unit cell. Symmetry conditions are applied to create a single unit cell result in order to conserve CPU-time. The full model results (right hand side of Figure 5.7) represent a $6 \times 6 \times 6$ mirroring, or replication, of the unit cell results. The array was made possible by a Matlab script called stı_gen_3d_array function.

The most consistent formation within each microstructure are the plates of material. The plates are strategically placed depending on the value and location of the two equal stress states. If the equal stress values are larger than the third stress, a solid plate of unknown thickness is formed on the plane of where those stresses are. The figures exemplifying this scenario are 5.7(b), 5.7(f), 5.7(g) and 5.7(h). If the equal stress values are not zero and lower than the third stress, a solid plate is not formed and instead the material is distributed mostly on the other two planes. Figures 5.7(d) and 5.7(e) match this scenario and contain many regions of hollow or void material.

For cases with a dominant load direction, fibrous microstructures provide the optimal geometry. Figure 5.7(g) portrays exactly that with hollow fibers in the xplane. A slightly different scenario involves uni-axial stress, where two or three stress states are zero. There lies uni-axial stress in the $x$-direction of Figure 5.7(a), which explains the single fibers in each array along with no other supporting material.

\subsubsection{Composite Microstructures}

Similar to the cases discussed above for porous microstructures, this section will focus on composite microstructures and slightly different conditions. The stress 
states, number of iterations and volume fraction all remain the same for each case, however, the domain consists of a two phase composite in which the stiffer phase has a Young's Modulus of unity and the lower stiffness phase has aYoung's Modulus of 0.5. . Table 5.4 shows the applied stress statescorresponding to the microstructures in Figure 5.8.

\begin{tabular}{|c|c|c|c|}
\hline Figure 5.8( ) & $\boldsymbol{\sigma}_{\mathbf{x}}$ & $\boldsymbol{\sigma}_{\mathbf{y}}$ & $\boldsymbol{\sigma}_{\mathbf{z}}$ \\
\hline $\mathrm{a}$ & 1 & 0 & 0 \\
\hline $\mathrm{b}$ & 1 & 1 & 0 \\
\hline $\mathrm{c}$ & 1 & -1 & -1 \\
\hline $\mathrm{d}$ & 1 & 0.5 & 0.5 \\
\hline $\mathrm{e}$ & 1 & 0.75 & 0.75 \\
\hline $\mathrm{f}$ & 1 & 1.25 & 1.25 \\
\hline $\mathrm{g}$ & 1 & 1.5 & 1.5 \\
\hline
\end{tabular}

Table 5.4: Stress values for each composite microstructure figure

(a)
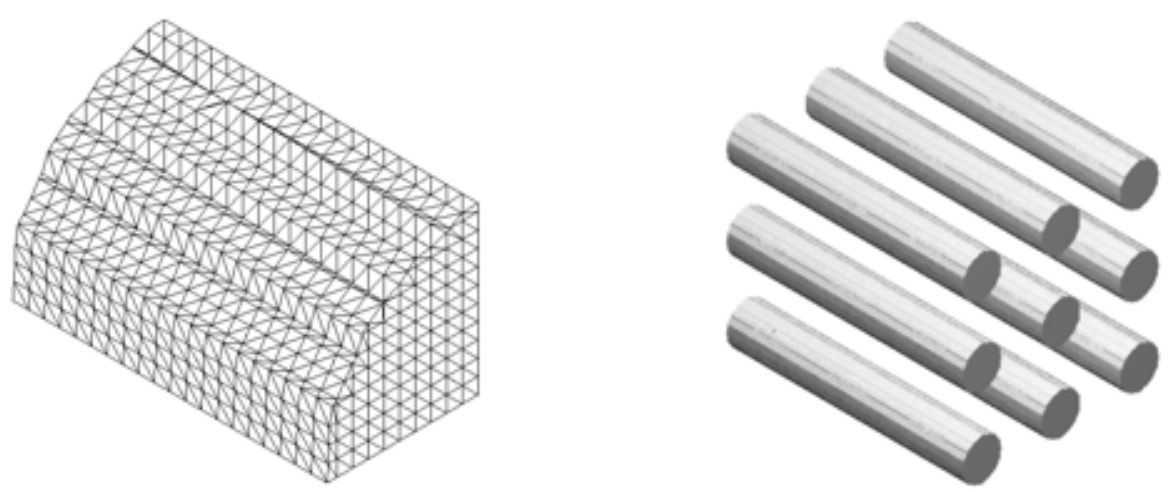
(b)

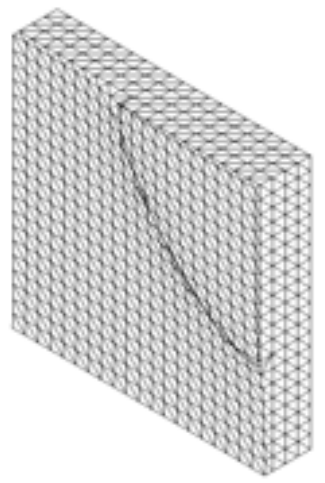

(c)

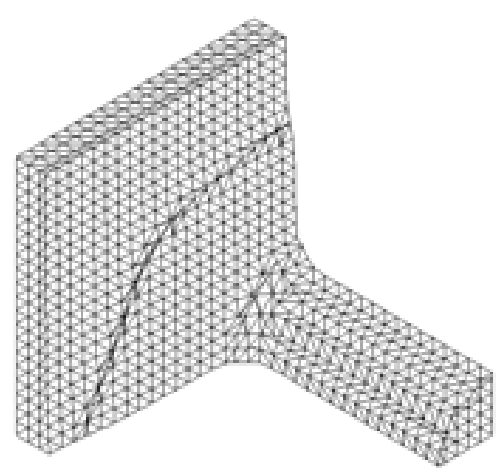

(d)

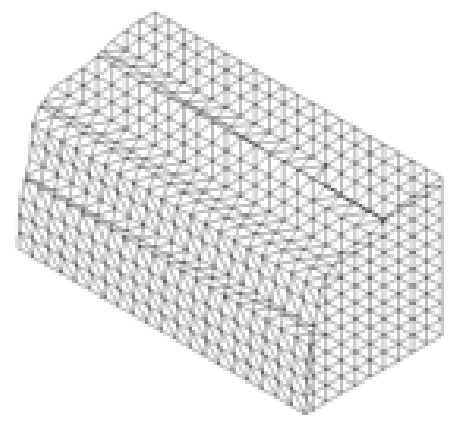

(e)

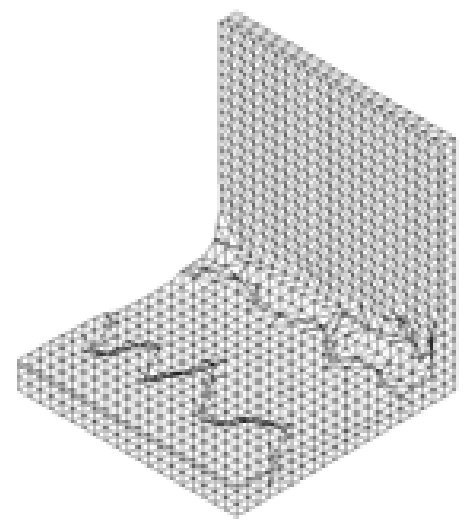

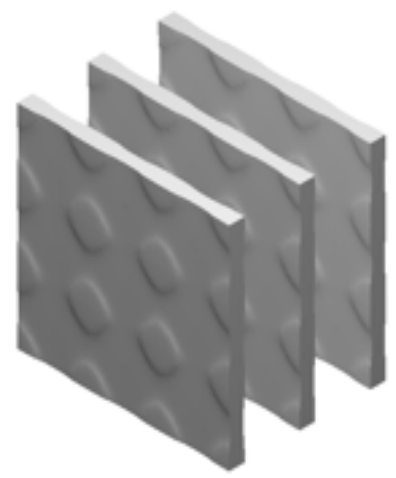
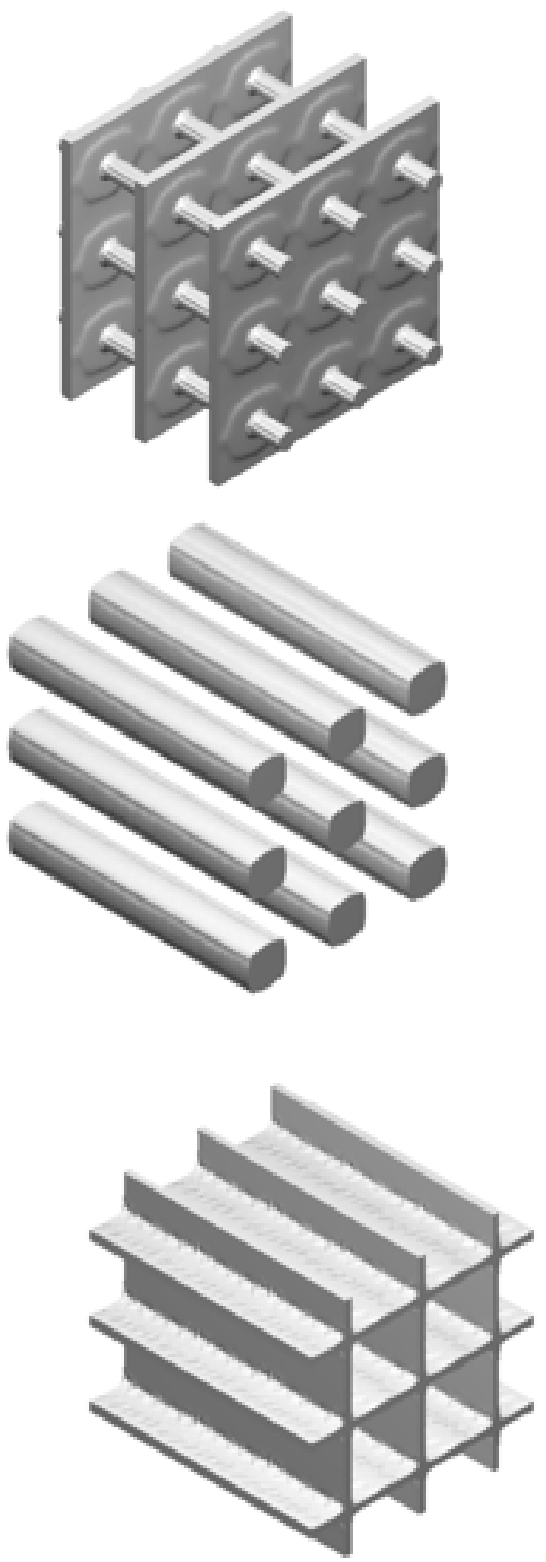
(f)
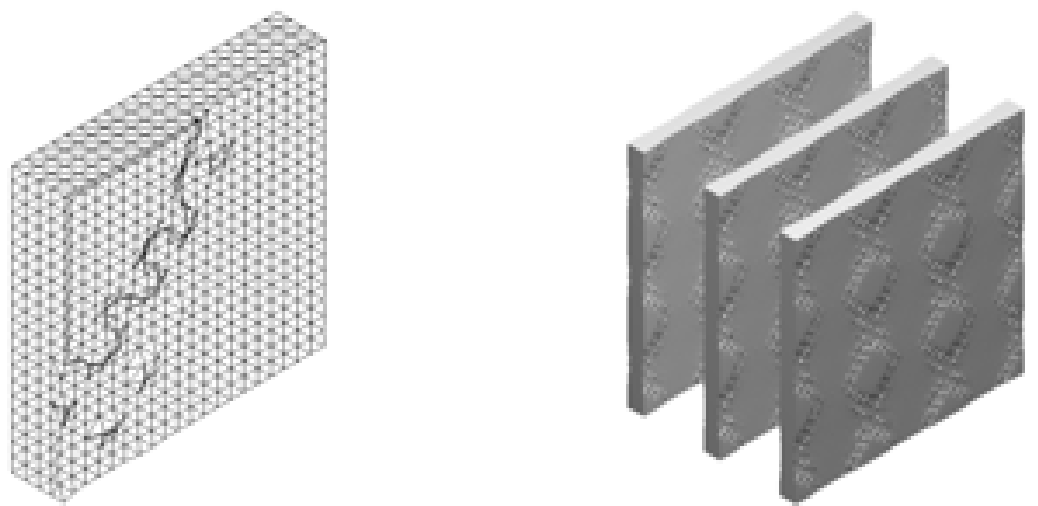

$(\mathrm{g})$
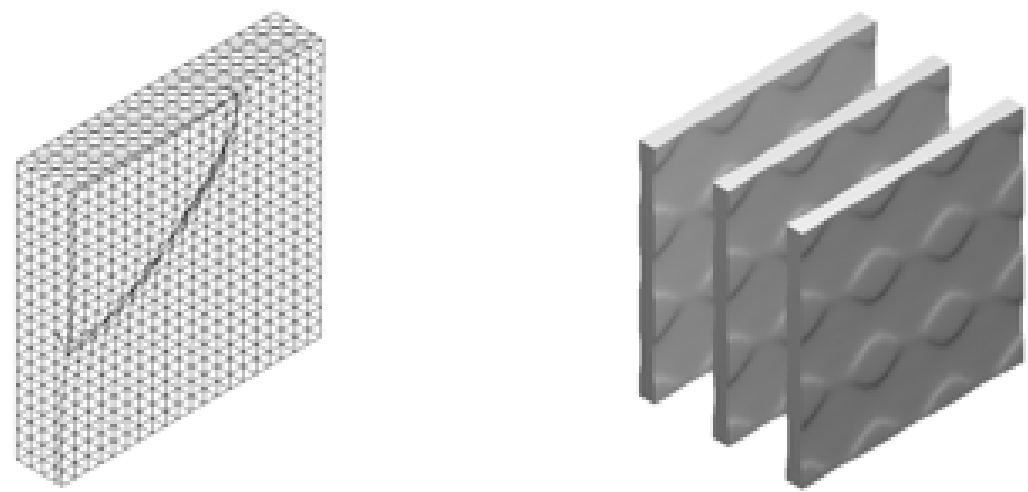

Figure 5.8: Two-phase composite microstructures; (a) through (g) cases correspond to conditions in Table 5.4

Similar comparisons and analysis can be for to the composites as with the porous microstructures. In some cases, the optimal topology takes on a different form due to the matrix material's ability to carry a fraction of the load. For example, in Figure 5.7(f), for the porous materials, fibrous regions carry the stress in the $x$ direction while in the corresponding composite case, the fibers do not appear because the matrix material is able to carry that stress.

To demonstrate the capability of creating physical models using 3-D printing technology, the STL files associated with the geometries shown in Figures 5.7(c) and 5.7(d) were used to manufacture physical models of these microstructures. These 
models were manufactured on a Dimension Model SST 3-D Printer. Photographs of these models are shown in Figure 5.9 and 5.10.

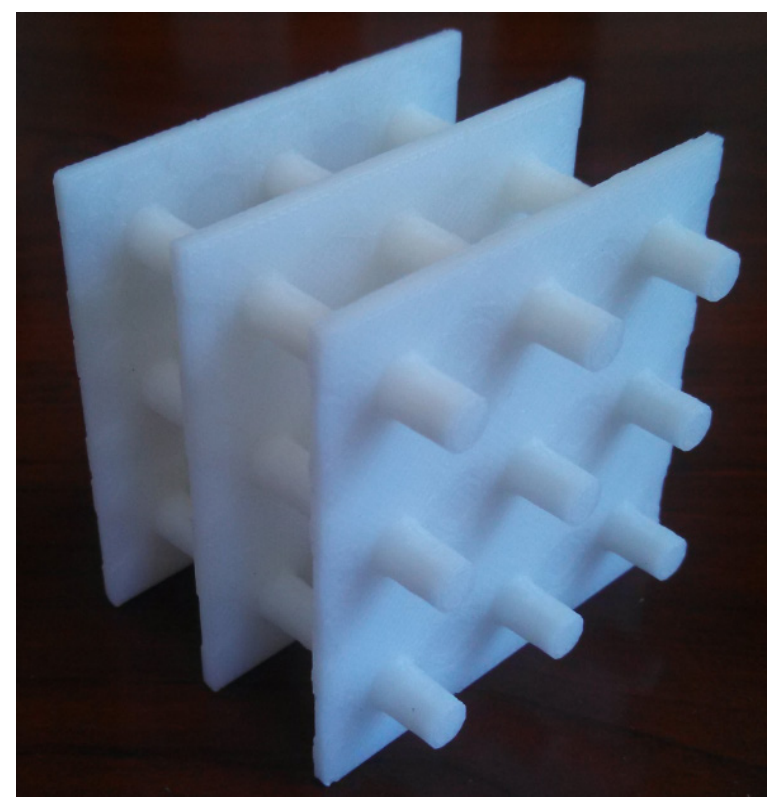

Figure 5.9: 3-D printed physical model of case shown in Figure 5.7(c)

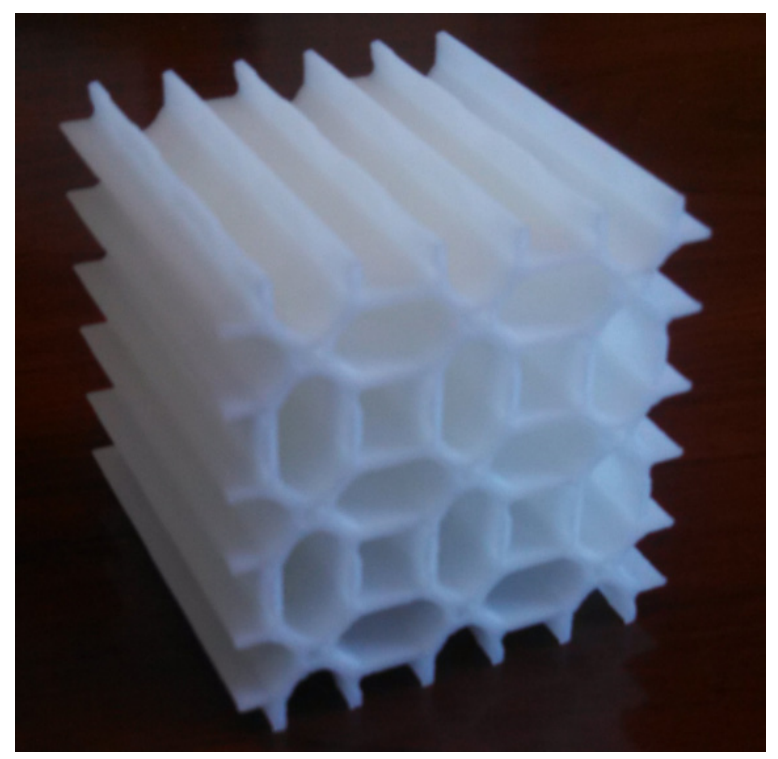

Figure 5.10: 3-D printed physical model of case shown in Figure 5.7(d) 


\section{CHAPTER 6}

\section{CONCLUSIONS AND FUTURE WORK}

Due to economic and environmental reasons, the interest in topology optimization and minimum weight structures has increased significantly in recent years. The PMR scheme as implemented in the Matlab script provides an effective tool for the identification of optimized structures; as well as graphically representing them in an external CAD program or for manufacturing with an additive manufacturing process.

The Matlab script developed in this research provides a self-contained code in which the user can easily define a general 3-D topology optimization problem and the code output includes an STL model file that represents the optimized topology. Several test cases are presented which confirm the ability of the code to successfully identify the known optimal topology for a particular structure. Future work could include more exhaustive validations of the code for other load configurations. The second set of test cases involved the determination of optimal microstructures for porous and two-phase composite materials. While the results obtained appear to be qualitatively reasonable, future work should develop more rigorous validation cases

for which the optimal microstructure can be established either analytically or through some other optimization procedure. Such analyses are required to confirm whether the PMR scheme can accurately identify optimal microstructures for porous or composite materials. 
This work included significant effort to optimize the computational efficiency of the code, particularly through imposition of symmetry conditions and the implementation of an efficient element assembly method. Although attempts were made to improve the efficiency of the Matlab solver in solving the global equations, the default solver as provided by the Matlab "backslash" equation solver proved to be the most efficient. Future work, however, should include further efforts to improve computational efficiency, perhaps through the use of iterative equations solvers and/or parallelization of parts of the code for efficient use of computers with parallel processing capabilities.

Finally, after thorough validation of the PMR code for the optimization of multi-phase composites, this method has the potential to provide an excellent tool for exploring methods for optimizing the local microstructure of structures. The microstructure of traditional composites is constrained by available manufacturing methods and the resulting configuration of reinforcing and matrix phases. With the advent of additive manufacturing methods where multiple phases can be deposited in any imaginable configuration, opportunities exist to locally tailor the microstructure based on the local stress state. Design tools such as the PMR optimization method, coupled with emerging manufacturing methods, could lead to a new generation of lightweight and strong materials. 
APPENDIX A

\section{SYMMETRY TEST CASES}

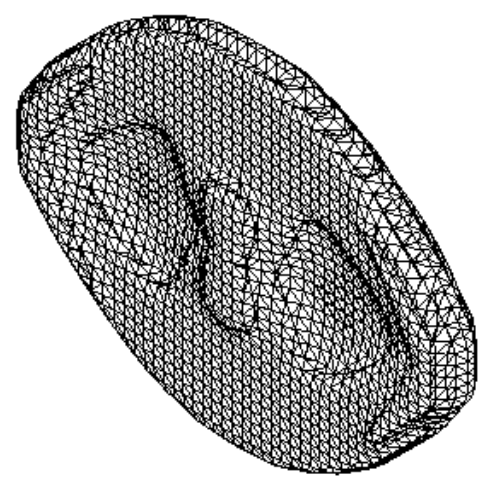

Figure A.1: Michell-Arch results using no symmetry for Case 1

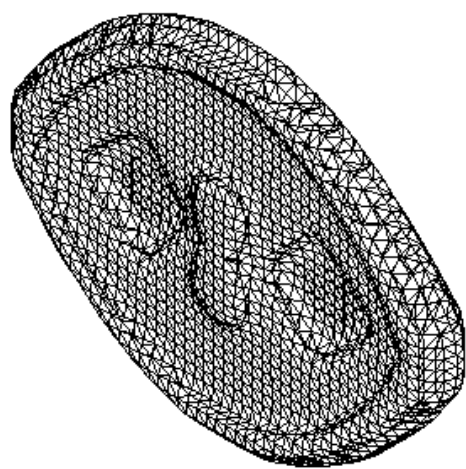

Figure A.2: Michell-Arch results using x-symmetry for Case 2

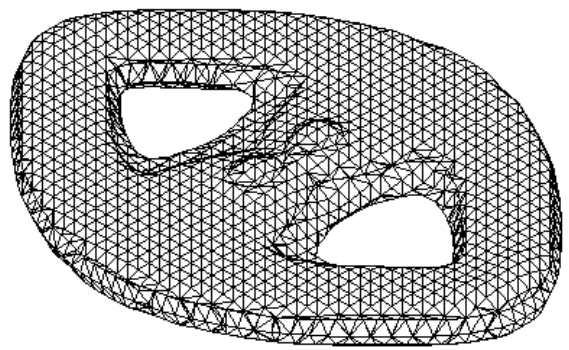

Figure A.3: Michell-Arch results using y-symmetry for Case 3 


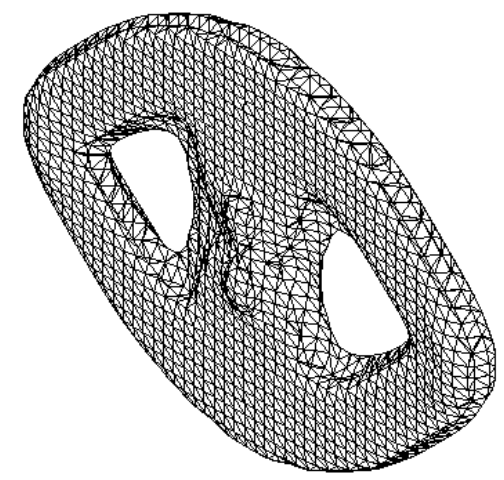

Figure A.4: Michell-Arch results using z-symmetry for Case 4

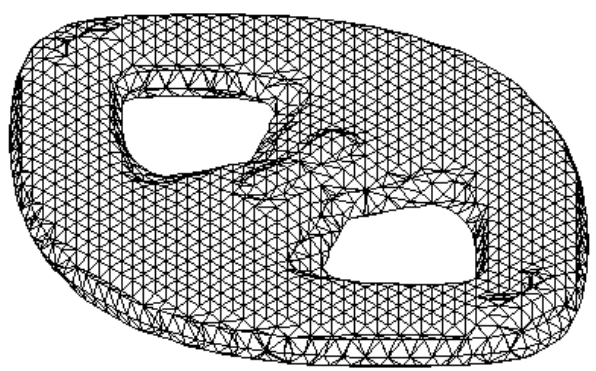

Figure A.5: Michell-Arch results using xy-symmetry for Case 5

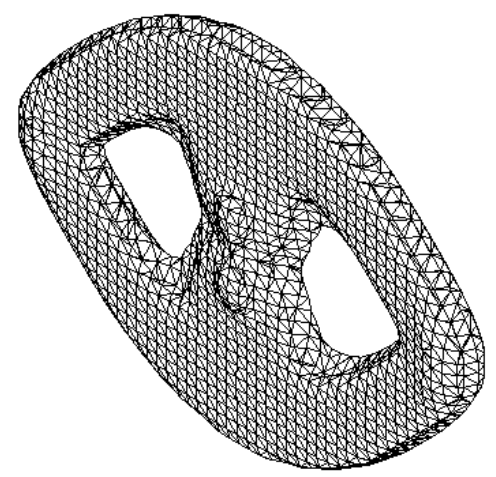

Figure A.6: Michell-Arch results using zx-symmetry for Case 6

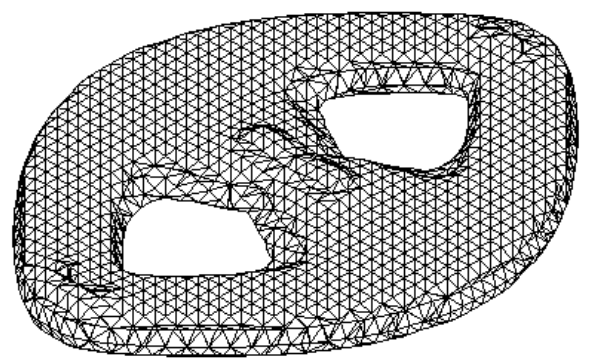

Figure A.7: Michell-Arch results using yz-symmetry for Case 7 


\section{APPENDIX B \\ MATLAB SCRIPT FOR SYMMETRY CASES}

The Matlab script for the Michell-arch symmetry cases is given below. Upon running the script, the user will be asked to specify which cases to run. By entering 1, all of the cases will run sequentially. If a single case is desired, the user may enter the case number manually when asked.

Within the loop of each case, the parameters are initialized and defined appropriately depending on the geometrical orientation. These parameters include nelx, nely, nelz, node_type, volfrac, iter, dbc, forces, sym, and constraint. After the loop is completed, the PMR_3D main script is called and the results and appropriate symmetry conditions are imposed to generate an STL file for viewing in an outside program such as 3D Myriad Reader.

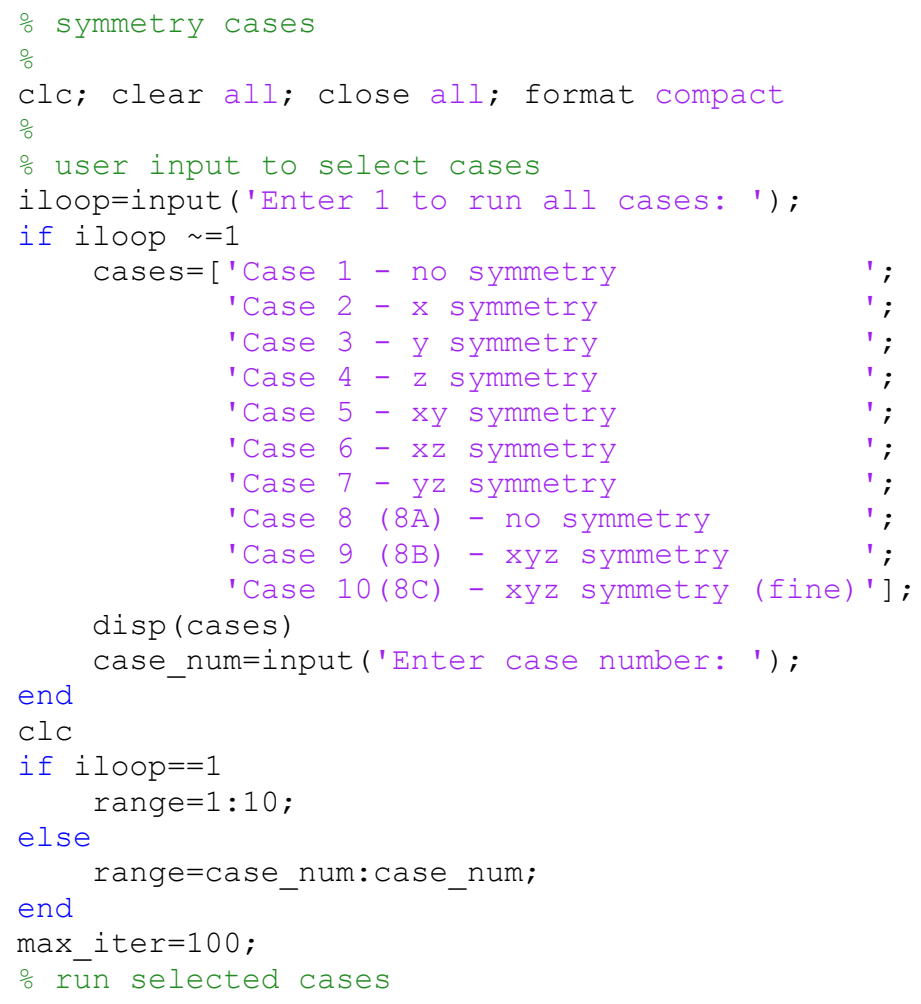




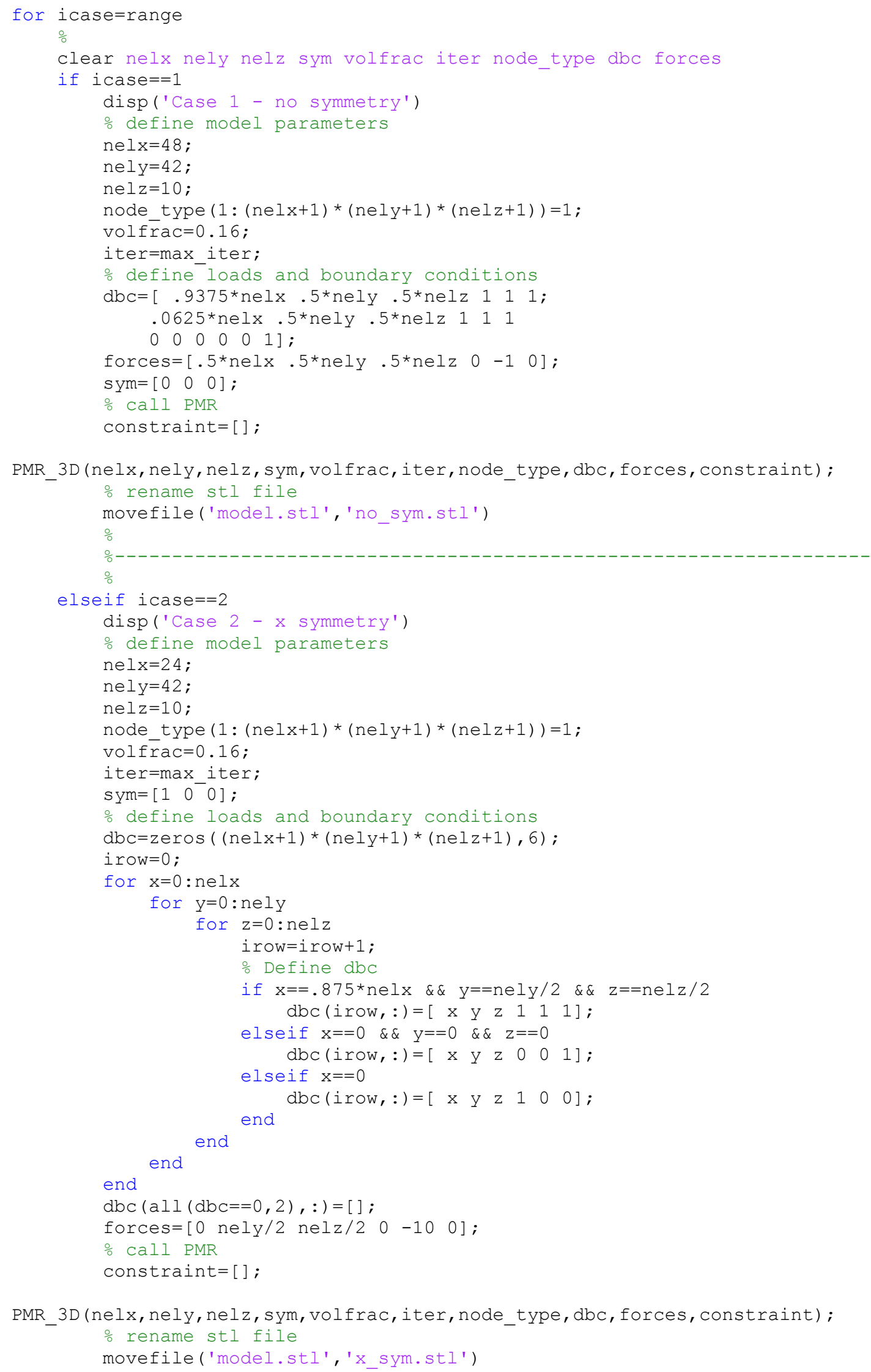

PMR_3D (nelx, nely, nelz, sym, volfrac,iter, node_type, dbc, forces, constraint); o rename stl file 


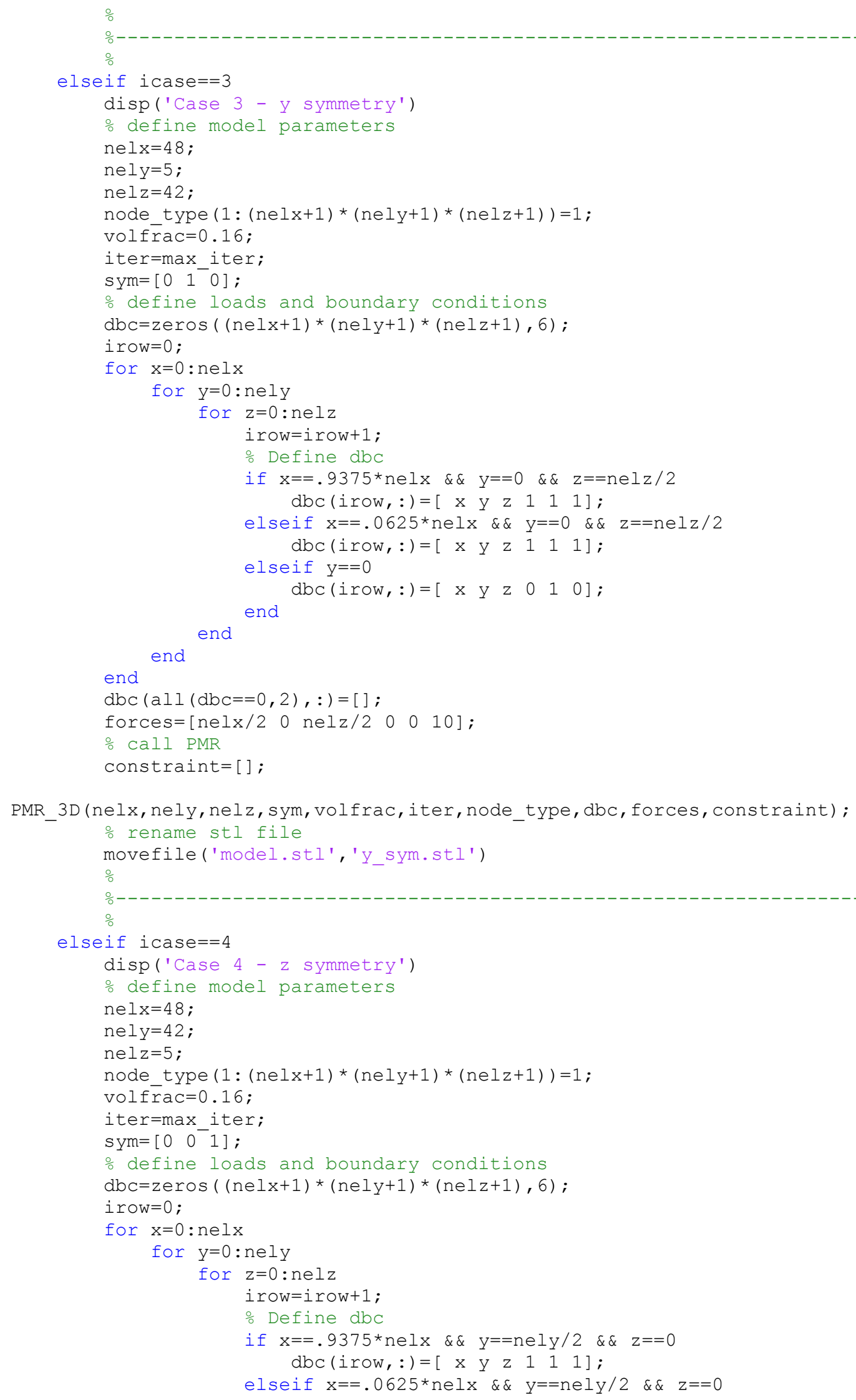




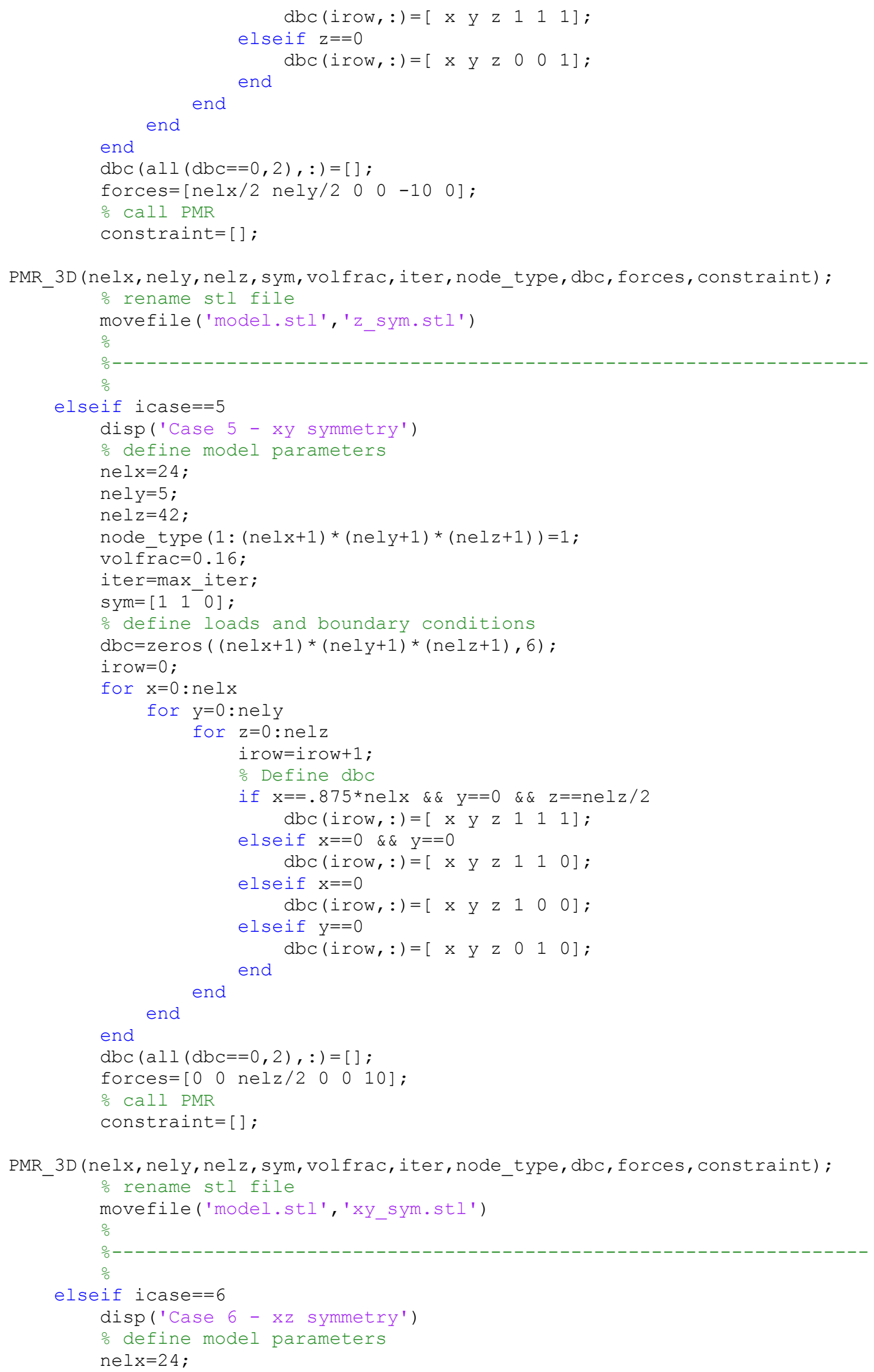




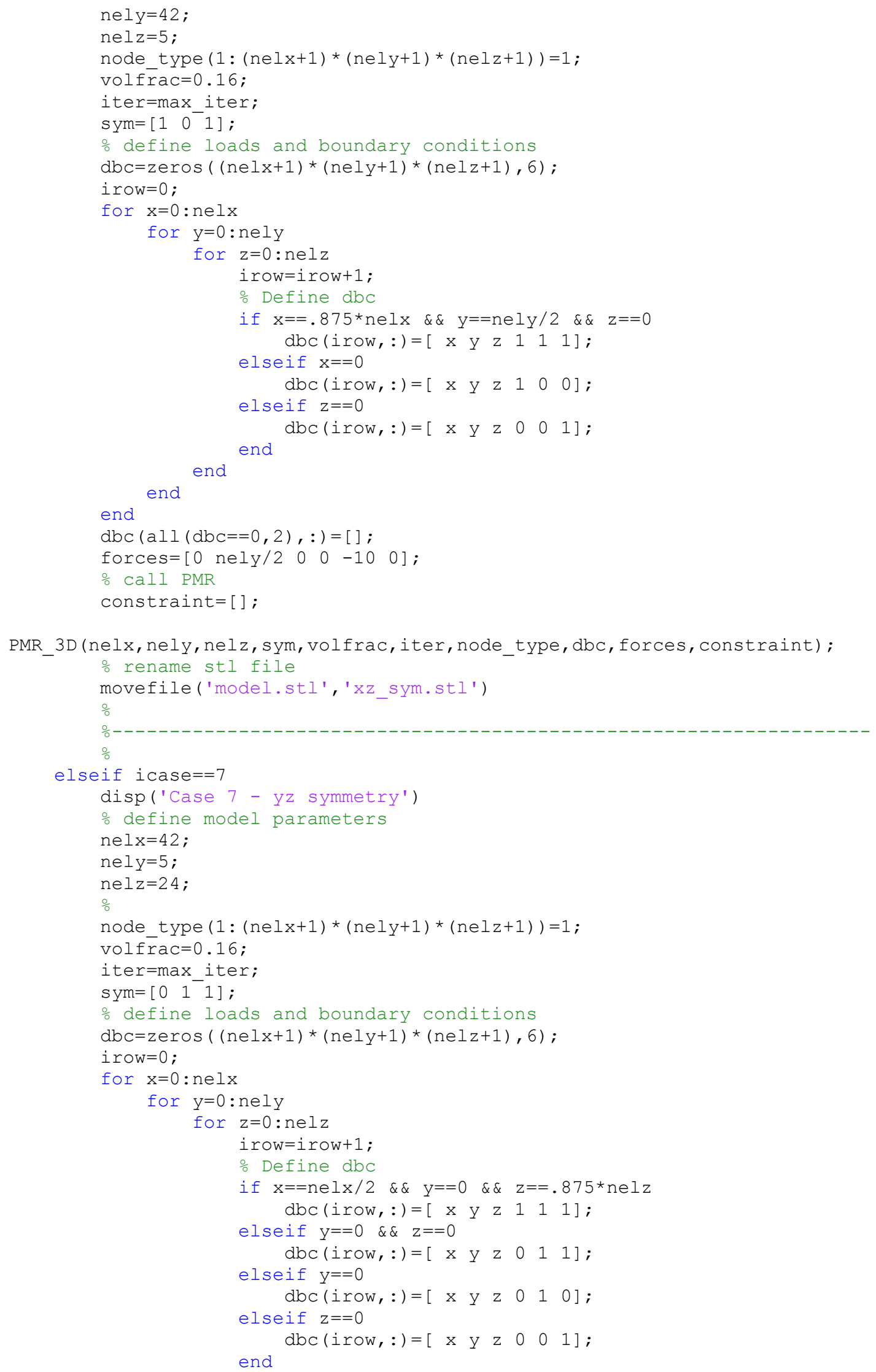




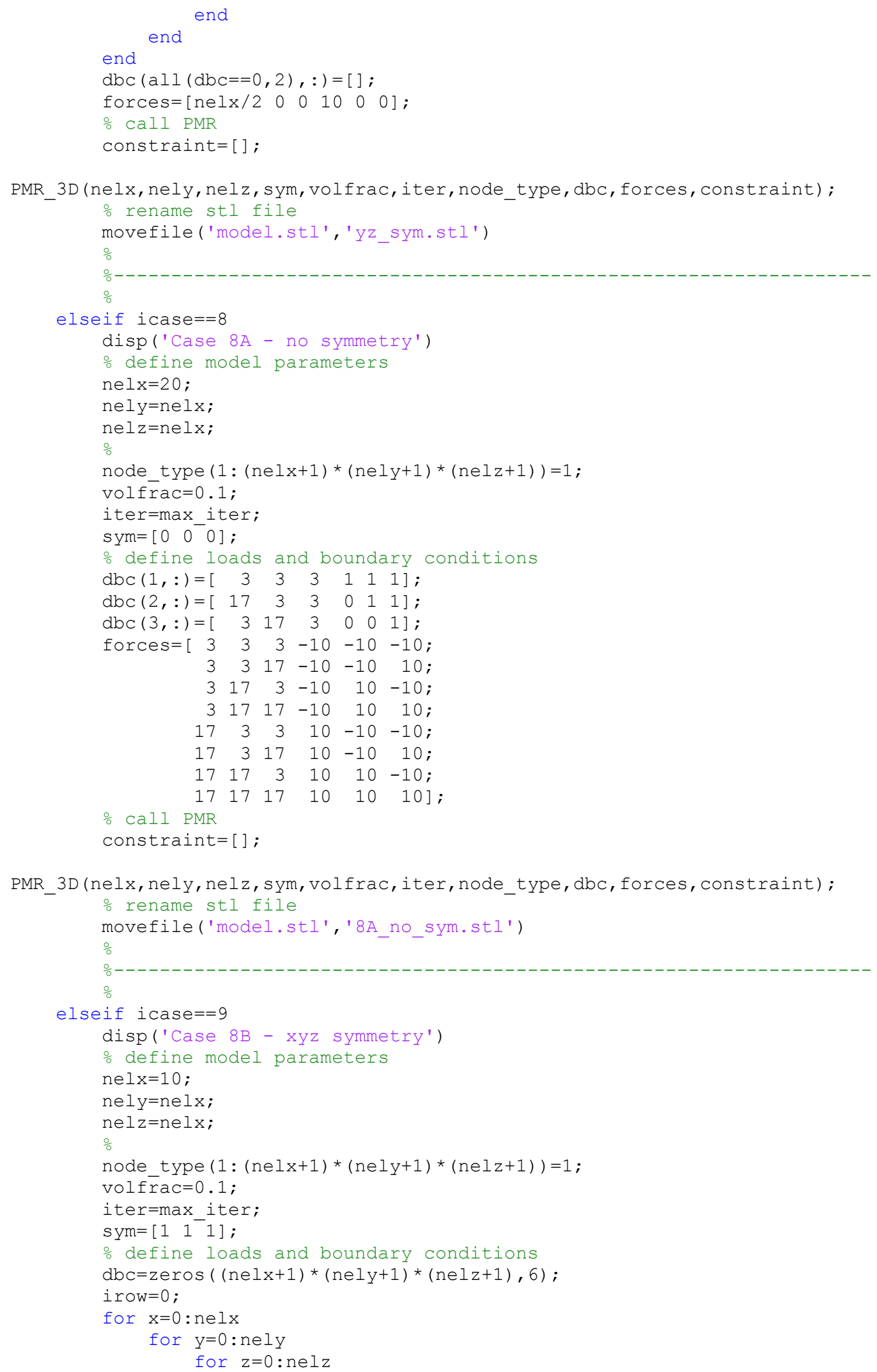




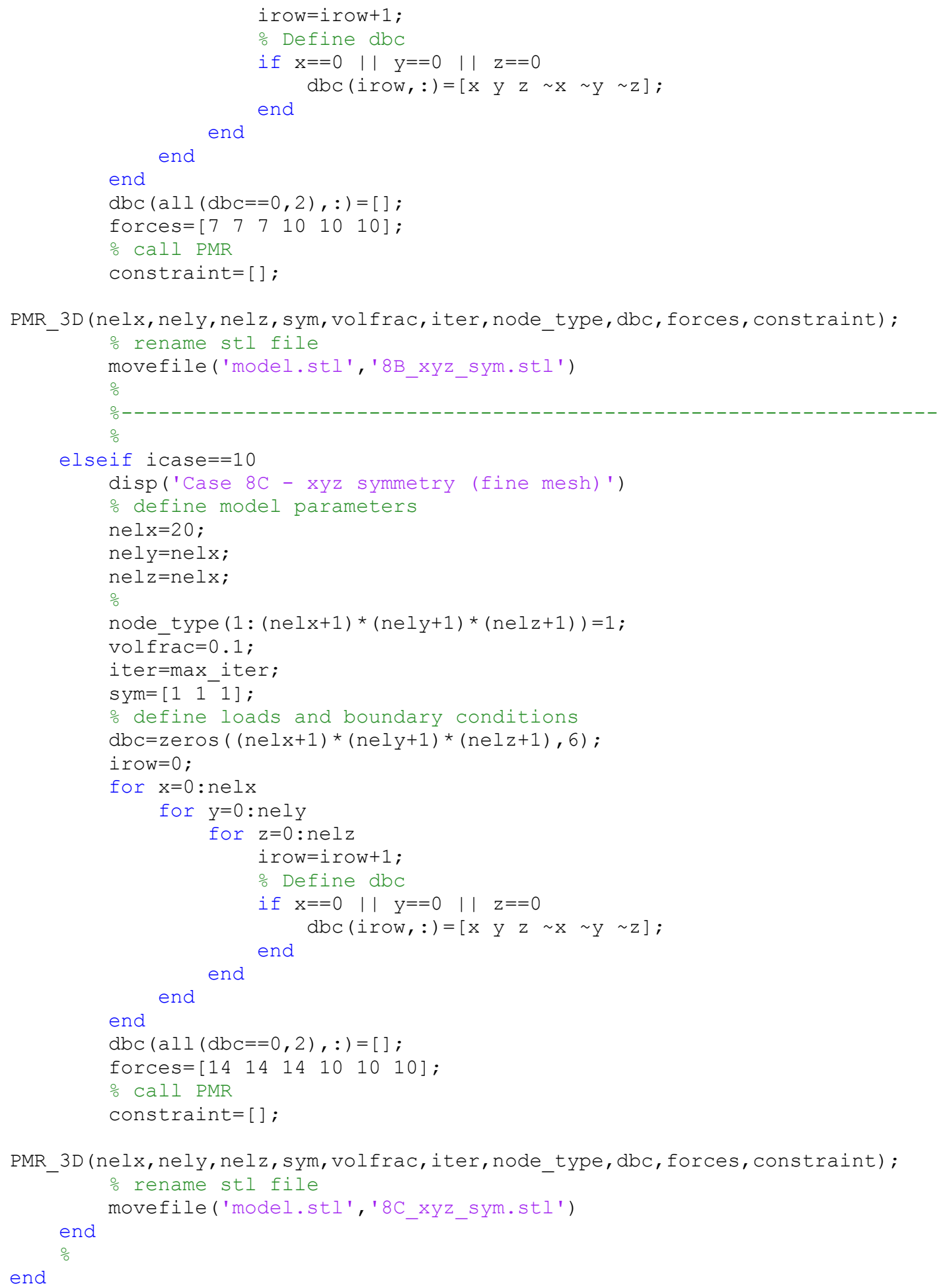




\section{BIBLIOGRAPHY}

[1] Taggart, D. G., Dewhurst, P., Dobrot, L., and Gill, D., "Development of a Beta Function Based Topology Optimization Procedure,” 2008 Abaqus Users Conference, Newport, RI, May 2008.

[2] Taggart, D. G. and Dewhurst, P., "A Novel Numerical Topology Optimization Method," 8th World Congress on Computational Mechanics and 5th European Congress on Computational Methods in Applied Sciences and Engineering, July 2008 .

[3] Taggart, D. G., Jahns, D., Nair, A. and Dewhurst, P., "Application of the PMR Topology Optimization Scheme to Dual Material Structures," ASME 2010 International Mechanical Engineering Congress and Exposition, Vancouver, Canada, November 2010.

[4] Taggart, D. G. and Dewhurst, P., "Development and Validation of a Numerical Topology Optimization Scheme for Two and Three Dimensional Structures," Advances in Engineering Software, 41, 910-915, 2010.

[5] Taggart, D. G., Dewhurst, P. and Nair, A. "System and Methods for Finite Element Based Topology Optimization”, US. Patent 8.335.668, 2012.

[6] Wilde, D.J. and Beightler, C.S., Foundations of Optimization, Prentice-Hall, Inc., 1967.

[7] Michell, A.G.M. LVIII. "The Limits of Economy of Material in FrameStructures," The London, Edinburgh, and Dublin Philosophical Magazine and Journal of Science, 8(47):589-597, 1904.

[8] Schmit, L.A., "Structural Design by Systematic Synthesis," Second Conference on Electronic Computation, ASCE, Pittsburg, 105-132, 1960.

[9] Bendsøe, M.P. and Sigmund, O., “Topology Optimization,” Springer-Verlag, 2004.

[10] Sant'Anna, Hervandil M., and Jun S.O. Fonseca. "Topology Optimization of Continuum Two-Dimensional Structures under Compliance and Stress Constraints," Tech. Vol. 21. Brazil: n.p., 2002. Print.

[11] Bendsøe, M.P., "Optimal shape design as a material distribution problem," Structural Optimization 1. 193-202. 1989. 
[12] Rozvany, G.I.N., Zhou, M. and Birker, T., "Generalized shape optimization without homogenization,” Structural Optimization 4. 250-254. 1992.

[13] Browne, Philip A. "Topology Optimization of Linear Elastic Structures." Thesis. University of Bath, 2013. Print.

[14] Aremu, A., I. Ashcroft, R. Hague, R. Wildman, and C. Tuck. Suitability of SIMP and BESO Topology Optimization Algorithms for Additive Manufacturing. Tech. Loughborough : n.p., 2010. Print.

[15] Sigmund, O., "A 99 line topology optimization code written in Matlab," Structural and Multidisciplinary Optimization, Volume 21 Issue 2, 120-127, 2001.

[16] Xie, Y.M. and Steven G.P., Evolutionary Structural Optimization, SpringerVerlag, Berlin, 1997.

[17] Xie, Mike. "Evolutionary Structural Optimization." Innovative Structures and Materials. RMIT, n.d. Web. 1 Mar. 2014.

$<$ http://www.rmit.edu.au/browse/Research\%2FInstitutes,\%20Centres\%20and $\% 20$ Groups $\% 2$ FCentres $\% 2$ FInnovative $\% 20$ Structures $\% 20$ and $\% 20$ Materials $\%$ 2FProjects\%2FEvolutionary\%20Structural\%20Optimisation/>

[18] O.M. Querin, G.P. Steven, Y.M. Xie, Evolutionary structural optimization (ESO) using a bidirectional algorithm, Engineering Computations, Vol. 15 Iss: 8, pp.1031 - 1048. 1998.

[19] Boer, Edward D. Topology and Shape Optimization for Non-Linear Problems. Tech. Sydney: n.p., 2010. Print.

[20] Diaz, A. and Sigmund, O., "Checkerboard layouts in pattern optimization," Structural Optimization. 10:40-45, 1995.

[21] Bendsoe, M.P., Diaz, A., Kikuchi, N., "Topology and generalized layout optimization of elastic structures, ” Topology design of structures. pp.159 205. 1993.

[22] Sigmund, O. Design of Material Structures using Topology Optimization. Ph. D. Thesis, Department of Solid Mechanics, Technical University of Denmark. 1994. 
[23] Shukla, Avinash, Anadi Misra, and Sunil Kumar. Checkerboard Problem in Finite Element Based Topology Optimization. Publication no. 22311963. 4th ed. Vol. 6. India: n.p., 2013. Print. IJAET.

[24] Jairo A. Martins and István Kövesdy (2012). Overview in the Application of FEM in Mining and the Study of Case: Stress Analysis in Pulleys of StackerReclaimers: FEM vs. Analytical, Finite Element Analysis - Applications in Mechanical Engineering, Dr. Farzad Ebrahimi (Ed.), ISBN: 978-953-51-07170, InTech, DOI: 10.5772/46165. Available from:

$<$ http://www.intechopen.com/books/finite-element-analysis-applications-inmechanical-engineering/overview-in-the-application-of-fem-in-mining-andthe-study-of-case-stress-analysis-in-pulleys-of-sta> .

[25] Perumal, Logah, and Daw Thet Thet Mon. Finite Elements for Engineering Analysis: A Brief Review. Tech. Vol. 10. Singapore: IACSIT, 2011. Print.

[26] Logan, D.L., A First Course in the Finite Element Method, 5th ed. Stamford, CT: Cengage Learning, 2011.

[27] Gavin, Henri P. "Mathematical Properties of Stiffness Matrices." Duke University, 9 Dec. 2013. Web.

[28] Ortiz-Bernardin, Alejandro. "An Efficient Finite Element Assembly in Matlab." IMechanica. N.p., n.d. Web. 22 Dec. 2013.

$<$ http://www.imechanica.org/node/7552>.

[29] "MYRIAD 3D Reader Download." N.p., n.d. Web. 04 Mar. 2014. $<$ http://www.myriadviewer.com/myriad-3d-reader-download $>$. 